CAFE MOCHA: An Integrated Platform for Discovering Clinically Relevant Molecular

Changes in Cancer; an Example of Distant Metastasis and Recurrence-linked

\title{
Classifiers in Head and Neck Squamous Cell Carcinoma
}

Neeraja M Krishnan ${ }^{1}$, Mohanraj ${ }^{1}$, Janani Hariharan ${ }^{1}$, and Binay Panda ${ }^{1,2^{*}}$

8 Bangalore, Karnataka 560100, India

$9 \quad{ }^{2}$ Strand Life Sciences, Bangalore, Karnataka 560024, India

*To whom correspondence should be addressed. Email: binay@ganitlabs.in

\section{Abstract}

\section{Background}

CAFE MOCHA (Clinical Association of Functionally Established MOlecular CHAnges) is

an integrated GUI-driven computational and statistical framework to discover molecular signatures linked to a specific clinical attribute in a cancer type. We tested CAFE MOCHA in head and neck squamous cell carcinoma (HNSCC) for discovering a signature linked to distant metastasis and recurrence (MR) in 517 tumors from TCGA and validated the signature in 18 tumors from an independent cohort.

\section{Methods}

21 The platform integrates mutations and indels, gene expression, DNA methylation and copy number variations to discover a classifier first, predict an incoming tumour for the same by

23 pulling defined class variables into a single framework that incorporates a coordinate geometry-based algorithm, called Complete Specificity Margin Based Clustering (CSMBC) with $100 \%$ specificity. CAFE MOCHA classifies an incoming tumour sample using either a 
27 deployed using the install $4 j$ multi-platform installer.

\section{Results}

29 We tested CAFE MOCHA to discover a signature for distant metastasis and recurrence in

30 HNSCC. The signature MR44 in HNSCC yielded $80 \%$ sensitivity and $100 \%$ specificity in

31 the discovery stage and $100 \%$ sensitivity and $100 \%$ specificity in the validation stage.

\section{Conclusions}

33 CAFE MOCHA is a cancer type- and clinical attribute-agnostic computational and

34 statistical framework to discover integrated molecular signature for a specific clinical

35 attribute.

36

37 CAFE MOCHA is available in GitHub (https://github.com/binaypanda/CAFEMOCHA).

38

39 Key Words: mutation, methylation, gene expression, copy number variation (CNV),

40 sensitivity, specificity and integrated platform.

41

42

43 


\section{Introduction}

Cancer progression is linked to molecular changes at multiple levels, such as somatic mutations, gene expression, DNA methylation and copy number changes. In the last 5yrs, a large amount of data on key variants in multiple cancers has been generated by international consortia, like The Cancer Genome Atlas (TCGA) (Editorial, 2015; Weinstein et al, 2013), International Cancer Genome Consortium (ICGC) (Hudson et al,

512010 ) and individual laboratories, aiding our understanding of various cancers at molecular level. Utilizing the vast amount of molecular data from studies on tumour genomes, exomes, transcriptomes and methylomes, and linking those with data from genetic and

54 functional studies will help find clinically relevant insights. Although there are existing databases and studies that combine molecular changes across cancer types (Deng et al, 2016; Huang et al, 2015; Netanely et al, 2016), studies linking the events explicitly within the same tumour type across a large number of samples to predict signatures for a specific clinical attribute are currently lacking.

Here, we describe a cancer type-agnostic computational and statistical framework with a user-friendly graphical-user-interface (GUI) to discover integrated signatures using

61 six tumour-specific event types; somatic mutations and indels (mut), DNA copy number changes (cnv), gene expression changes (expr), 5-cytosine DNA methylation changes (meth), functional copy number changes (fcnv, where CNVs are linked to gene expression changes) and functional cis-regulatory DNA methylation changes (fmeth, where hyperand hypo-methylation result in down- and up-regulation of effector gene expression respectively). CAFE MOCHA, in addition to classifying categorical events like mut and cnv, uses an algorithm called Complete Specificity Margin Based Clustering (CSMBC) to classify quantitative (expr and meth) and coupled events (fmeth and fcnv), and combines 
signatures describing the clinical variable with $100 \%$ specificity. We tested CAFE MOCHA

to discover a signature linked with distant metastasis and recurrence in head and neck

squamous cell carcinoma (HNSCC) using 434 tumours from TCGA as a discovery cohort,

which was validated in an independent cohort of 18 tumours. The integrated signature

MR44 for metastatic and recurrent tumours in HNSCC (MR44) yielded a sensitivity of

$79.52 \%$ and a specificity of $100 \%$ in the discovery cohort and with $100 \%$ sensitivity and $100 \%$ specificity in the validation cohort.

\section{Materials and Methods}

Discovery module

MOCHA has two independent modules, discovery and prediction. In the discovery module, under mut. A matrix of following values (thresholds) were considered for cnvs; -2: full copy deletion, -1: allelic deletion, 1: low-copy amplification, 2: high-copy amplification, and 0: lack of any copy number variation. For expression and methylation, RSEM (Li \& Dewey, 2011) -normalized gene-wise intensity matrix and a probe-wise matrix of pre-processed $\beta$ values (from Illumina 450k whole-genome methylation arrays), respectively, were used. The methylation data was pre-processed, including normalization and batch correction, as described previously (Krishnan et al, 2016). Tumour samples assayed for all the four events (mut, cnv, expr and meth) were considered for the discovery phase. The perturbed genes were passed through a functional filter (detailed below) before being used in the integrated analysis. In the case of methylation data, a sub-region filter was applied and only probes located in the gene promoters, transcription start site (TSS200 and TSS1500) 
chosen clinical attributes with complete specificity. Quantitative events such as expression

(expr) and methylation (meth) were linked to clinical attributes using an algorithm called

97 Complete Specificity Margin Based Clustering (CSMBC) algorithm (detailed below). A somatic filter based on an unpaired $t$-test $(P<0.05)$ was further used to retain only tumour-specific expr and meth events. Expression events were further coupled with meth and cnvs to generate coupled events (fmeth and fcnv) respectively, where meth and cnvs affected the target gene expression. We only considered hyper- and hypo-methylation events linked with the down- and up-regulation of downstream gene expression respectively.

\section{Data integration and making of the final signature}

Using the 'integrate' feature, the individually discovered events were combined into an integrated signature with two different filters (Figure 1B). In the first, a tumour-specific sample frequency-based filter agnostic to any event type was applied. In the second, all

event types' priorities were determined by detection sensitivity first and then those events that yield highest priority followed by highest sample frequency were chosen. These two

filters work contrarily, one retaining the high sample frequency quantitative and semiquantitative event types (expr, meth, fmeth, fcnv) and another the lower-sample frequency categorical and semi-categorical event types (mut, cnv, fcnv). Selected events were considered for removal of false positives using samples lacking complete cross-platform overlap from TCGA and the independently provided datasets (used for confirmation in the second stage of the discovery stage). The number of confirmed events was further

117 minimized using the sample frequency-based filter to constitute the discovery panel for 118 that specific clinical attribute. This minimal signature is then subjected to an independent validation using tumours assayed for all the four events (mut, cnv, expr, meth) (Figure 1B). 
121 from KEGG (Kanehisa et al, 2002; Kanehisa et al, 2004; Kanehisa et al, 2012)

122 (hsa05200).

CAFE MOCHA Interface

The interface for CAFE MOCHA was developed and deployed using Netbeans IDE $v$

8.1 (https://netbeans.org/downloads/). The Graphical User Interface (GUI) was designed

using JAVA AWT (http://www.javatpoint.com/java-awt) with SWING components

(http://www.javatpoint.com/java-swing) with its native OS GUI and appropriate controls.

The back end for this interface was built on a Linux-dependent platform with $R, B A S H$,

BEDTools (v2.3) and PERL as added dependencies. CAFE MOCHA requires installations

of $R$ package dependencies (minfi v1.18.2 (Aryee et al, 2014), wateRmelon v1.16.0

(Pidsley et al, 2013), IlluminaHumanMethylation450kmanifest v0.4.0, randomForest

v4.6.12(Breiman, 2001), varSeIRF v0.7.5, pheatmap v1.0.8, gplots v3.0.1, ggplot2 v2.1.0,

reshape 2 v1.4.1) to pre-process $450 \mathrm{k}$ idat files and generates the matrix of $\beta$ values. The platform provides the user with an option to install the dependencies. Under the discovery module, mut, cnv, expr and meth data can be browsed and acquired locally.

Functional filters

To ensure functional relevance of predicted tumour-specific molecular changes, we introduced various filters for different events to select genes of interest. We used IntOGen (Gundem et al, 2010) to select driver/potential driver mutations, MethHC (Huang et al, 2015) for known hyper- and hypomethylated genes in different cancer types, G2SBC

144 (Mosca et al, 2010) for selecting important differentially expressed genes associated with breast cancer, The Human Protein Atlas (Uhlen et al, 2015) for functional proteins, the dbDEPC 2.0 database (He et al, 2012) for manually curated differentially expressed 
147 proteins in human cancer and miRTarBase (Chou et al, 2016; Hsu et al, 2011; Hsu et al, 148 2014) for experimentally validated miRNA-target gene associations. In the case of micro149 RNA methylation, we considered those events where methylation of a microRNA 150 correlated with a concomitant expression change in one or more of its target gene(s). In 151 the case of fcnv, we considered where amplification and deletion in a gene was linked to 152 its up- and down-regulation respectively in tumour sample compared to normal. Only full 153 copy deletions (not allelic deletions) and amplifications were considered. Genes bearing 154 categorical events in at least one sample that passed the functional filter were considered 155 further

Complete Specificity Margin Based Clustering (CSMBC)

We devised a statistically framework, Complete Specificity Margin Based Clustering or CSMBC, for inferring the expression and the methylation boundaries of each cluster for a specific clinical attribute (Figure 2). In CSMBC, the number of clusters were fixed equivalent to the number of pre-defined clinical variables. For each cluster, axisperpendicular boundaries passing through the most extreme outliers on the expression/methylation coordinate axes were determined first. The number of overlaps was dependent upon the number of boundaries. In the simplest case, there can be two sub-categories. For example, metastasis as a category has two sub-categories, metastatic $(\mathrm{A})$; and non-metastatic $(\mathrm{B})$. In this simplest case, there can only be one overlap $(A \cap B)$. Likewise, if a clinical attribute has three (A, B and C) sub-categories (for example recurrent, non-recurrent, can't be defined) with four possible overlaps

$169(A \cap B, A \cap C, B \cap C, A \cap B \cap C)$. Using the above logic defined for categories and sub170 categories, all possible overlaps between clusters were identified first, and then the 171 minimum and maximum values for various overlaps were estimated according to the 172 following equations: 
174 For overlap between two sub-categories:

$\underset{i \neq j}{i} j_{\min }=\max (i, j) ; \underset{i \neq j}{i} j_{\max }=\min (i, j)$

177 For overlap between three sub-categories:

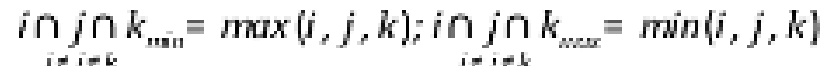

For overlap between four sub-categories:

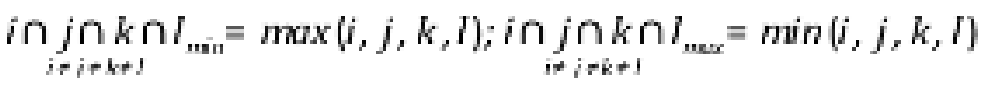

A sample was identified to be within $100 \%$ specificity margin of the cluster if it was not

in any region of overlap with other clusters (Figure 2). The total number of all such

samples, i.e., sensitivity of that cluster, was estimated according to the equation below:

For two sub-categories:

sensitivity $=n_{i}-n_{i}$;

For three sub-categories:

sensitivity $=n_{i}-n_{i n}-n_{i} n_{k}+n_{i n j n k}$

191 For four sub-categories:

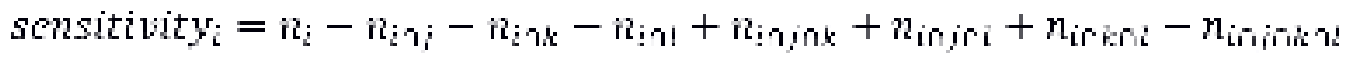

Finally, an unpaired $t$-test was performed between all tumour and all normal samples, tumour and normal groups were retained. 


\section{Prediction module}

The prediction module has two options: one, where the user provides somatic mutations/indels, tumour-specific expr, meth and cnv information, and second, where the user has somatic mutations/indel/cnv data but does not have expr and meth data from the matched normal samples. In the later case, CAFE MOCHA uses a built in normal database (all normal samples from the TCGA for a particular cancer type) to compute expression and methylation values for genes in control samples from RNASeq for expression and whole genome arrays (Illumina 450k) for DNA methylation. For the prediction module, the data is entered as per the pre-defined format (same as discovery panel) specific to a cancer type and clinical attribute of interest (Figure 1). Based on the user input, coupled events (fmeth and fcnv) are determined and used for the prediction module.

\section{Testing CAFE MOCHA to discover metastatic/recurrent HNSCC signature}

We tested CAFE MOCHA to discover integrated signature for metastatic and recurrent HNSCC tumours using tumours from TCGA dataset $(n=434)$, followed by confirmation (removal of false positives, the second stage of the discovery phase) using two sample cohorts, 42 samples from TCGA and 37 samples from an independent cohort (Krishnan et al, 2015; Krishnan et al, 2016), where the information on at least one of the event type out of the 4 events (mut, cnv, expr and meth) for the same tumour was available (Table 1, Supplementary Tables S1 and S2). Finally, the discovery panel was validated in 18 samples from an independent sample cohort (Krishnan et al, 2015; Krishnan et al, 2016), where all the four events were assayed for all the tumours (Supplementary Table S3). For the discovery module, we downloaded the Broad automated somatic mutations and indel call file, copy number data with gistic2 threshold, Illumina HiSeqV2 RSEM (7) -normalized RNA-seq gene expression data, 450k DNA methylation data and the clinical data from the 
224

UCSC Xena Browser (http://tinyurl.com/jhmg9b9). The distant metastasis (M1) and recurrent tumours (RFS_IND $=1$ ) were compared with non-metastatic $(\mathrm{M} 0)$ and nonrecurrent $($ RFS_IND $=0)$ tumours to derive a specific signature.

\section{Results}

Discovery of distant metastasis/recurrence-associated molecular signature MR44 in HNSCC

CAFE MOCHA identified a total of 6649 events from individual event type discovery (8 somatic mutations, 1436 cnvs (506 amplifications and 982 full copy deletions), 1098 expression-related events, 4018 methylation events, 27 fcnv events (8 high copy amplifications and 19 full copy deletions) and 62 fmeth events), associated with metastasis and recurrence in HNSCC. Integration across discovered events was performed using two filters as described in the Methods section. The filters resulted in 79 (22 expression-related events and 57 methylated genes) and 171 (7 mut, 84 cnvs (46 amplifications and 38 full copy deletions), 24 expr, 2 meth, 25 fcnv events (8 high copy amplifications and 17 full copy deletions) and 29 fmeth events) events, individually, and 232 (7 mut, 84 cnvs (46 amplifications and 38 full copy deletions), 30 expr, 57 meth, 25 fcnv events (8 high copy amplifications and 17 full copy deletions) and 29 fmeth events), cumulatively after removing any redundancy (Supplementary Table S4).

Using data at the second stage of the discovery phase (confirmation stage), 98 false positives events were eliminated. The remaining 134 events (Supplementary Figure S1) (5 mut, 83 cnvs (46 amplifications and 37 full copy deletions), 8 expr, 9 meth, 24 fcnv events (8 high copy amplifications and 16 full copy deletions) and 5 fmeth events) were further minimized to a final discovery panel of 44 events (MR44) that included 2 mut, 15 cnvs (3 amplifications and 12 full copy deletions), 8 expr, 9 meth, 8 fcnv events (5 high 
copy amplifications and 3 full copy deletions) and 2 fmeth events) (Figure 3A). The signature was further validated using an independent cohort of 18 samples (3 M1/R and 15 non-MR) of an independent oral tongue squamous cell carcinoma (OTSCC) cohort, with $100 \%$ sensitivity and $100 \%$ specificity. 17 out of the 44 events in MR44 signature were validated in at least one sample in the validation cohort (Figure 3B).

\section{Power of integration}

We wanted to investigate the power of integration (where all the six different event types were used to derive the signature) over lesser order combinations or with individual event type, in terms of the detection sensitivity and the number of events required to achieve maximum specificity. As shown in Figure 4, the detection sensitivity showed a gradual enhancement when the number of event types increased from one to six. For single-event analyses, meth and both mut and fmeth provided with a maximum and minimum sensitivity respectively $(37 \%, 7 \%$, Figure $4 \mathrm{~A})$. The number of $\mathrm{CNV}$ s required to achieve the highest possible level of sensitivity in a single-event analysis was the highest (83 events with $33 \%$ sensitivity). When using more than one event type, we obtained an increase in sensitivity to various levels gradually from a single-event to a six-events signature (Figure 4). The number of CNVs and both mut and fmeth required to get similar level of sensitivity was highest and lowest respectively for all combinations. Highest sensitivity $(80 \%)$ of detection was observed when all the six different event types were used to produce the integrated signature (Figure 4F). The impact of event integration on

271 detection sensitivity was observed to be greatest when the integration was performed in

272 the following order: meth + cnvs + expr + fmeth + fcnvs + mut. Each incremental

273 integration in this order caused a gradual increase in sensitivity $(37.35 \rightarrow 56.63 \rightarrow 68.67$

$274 \rightarrow 74.70 \rightarrow 77.11 \rightarrow 79.52$, Figure 4$)$. The actual events for all combinations and

275 individual event type analyses are illustrated in Supplementary Figure S1. 


\section{Discussion}

Integrating multiple molecular events is a strategy proposed to identify master

279 regulators in cancer (Gevaert \& Plevritis, 2013; Thingholm et al, 2016). However, a

280 meaningful integration should be incremental, with each level filtering out the unnecessary 281 and only retaining the functionally relevant associations. Furthermore, integration of data 282 should link a specific signature to clinically relevant tumour attributes in order for making 283 meaningful conclusions. Integrated analyses often fail to establish the link between

284 cancer-associated changes within the same tumour types across cohorts, perhaps due to 285 the low overlapping of clinical characteristics across cohorts and low reproducibility of 286 results across laboratories, geographies and discovery platforms. Additionally, data on 287 multiple events; genetic, transcriptomic and epigenetic changes, from the same sets of 288 tumour:matched normal samples are often not available, making it difficult to discover 289 truly integrated signatures. Nevertheless, such a discovery set, where all the events are 290 assayed for all tumour:normal pairs within the same cohort, is functionally more 291 meaningful. As multidimensionality increases with the availability of more data, especially 292 from the large consortia like TCGA and ICGC, it will become imperative to design and 293 implement easy-to-use and robust web-based platforms to discover (using a pre-defined 294 training set) multi-gene and multi-platform classifiers for a particular clinical attribute and 295 predict the same for an incoming new tumour sample. Currently, such a platform is lacking 296 that can discover tumour-specific genome-wide functional and somatic molecular changes 297 linked to a specific clinical attribute, using a sizeable multi-dimensional dataset. Keeping 298 this in mind, we devised CAFE MOCHA, an automated and integrated framework to 299 discover meaningful, functional and somatic molecular changes in a cancer type that links 300 the signature(s) to a specific clinical attribute. CAFE MOCHA is designed to use both user301 defined/generated and publicly available tumour and matched normal data. 
Additionally, the prediction module of CAFE MOCHA uses a backend database of

unmatched normal samples to increase the prediction scope of the tool for tumours where

matched normal samples are not available, especially where expression and methylation data is not available from matched normal samples. CAFE MOCHA is a cancer type- and clinical attribute-agnostic tool and has the ability to pull data on several event types under a single framework. CAFE MOCHA classifies events into three different types, categorical (mutations and CNV), quantitative (expression and methylation) and coupled/linked (functional CNVs and functional methylation) and uses an algorithm called complete specificity margin based clustering (CSMBC), a fully supervised approach, to identify clinically linked quantitative and coupled events. CSMBC is a modification of the Large Rectangle Margin Learning (LRML) approach described previously (kirmse \& Petersohn, 2011) and is conceptually similar to fast boxes which take the 'characterize' and then 'discriminate' approach of classification (Goh \& Rubin, 2014). The input for CSMBC is a continuous function spread across a single dimension, either expression or methylation.

For both these variables, an unsupervised clustering, which requires a priori knowledge of

318 the quantitative expression or methylation map, is not feasible since these are continuous 319 variables. The selection of boundary margins, according to the chosen clinical variables on the other hand does not require any prior knowledge of the quantitative spread of these events. Additionally, supervised clustering is faster than unsupervised clustering since it does not require iterative computing and does not require re-estimation of the cluster mean. Nevertheless, as pointed out in the past (Richards, 2013), a hybrid semi-supervised approach that uses the results of an unsupervised approach such as the $k$-means or Expectation Maximization (EM) as training areas for a supervised classification, might combine the advantages of both supervised and unsupervised classification approaches. 
classification sensitivity of a sample based on its proximity to the nearest boundary, would

further add confidence perhaps at the cost of sensitivity, particularly while predicting the clinical status of an incoming sample. While we plan to develop CAFE MOCHA taking these modifications into account in the future, the current implementation benefits from ensuring complete specificity for each clinical attribute-associated molecular event. One of the limitations of CSMBC is that its specificity is entirely dependent on the denominator, i.e., sample numbers and therefore, may be sub-optimal in a scenario where fewer numbers of tumour samples are present in the discovery set.

HNSCC are the sixth leading cause of cancer worldwide with 5-year survival of less than 50\% (Ferlay et al, 2010; Mishra \& Meherotra, 2014). Recent high-throughput studies employing computational methods have identified various genetic, transcriptomic and epigenetic changes from different subsites of HNSCC from different geographies (Agrawal et al, 2011; Cancer Genome Atlas, 2015; India Project Team of the International Cancer Genome, 2013; Krishnan et al, 2015; Pickering et al, 2013). Early-stage patients with HNSCC are usually treated using a single modality like surgery or radiotherapy whereas advanced-stage patients benefit from multi-modality therapies (Ridge et al, 2016). In head and neck tumours, identifying patients with tumours a priori that may undergo distant metastasis or loco-regional recurrence using primary-tumour-derived molecular signature will help manage patients better. CAFE MOCHA was tested using a robust dataset of 434 HNSCC tumours from TCGA where data on all four molecular events were available.

Despite the MR44 being an integrated signature, drawn from 83 metastatic/recurrent tumours with four different somatic events (mut, cnv, expr and meth) and two coupled events (fmeth and fcnv) available on all the tumours, the discovery sensitivity did not attain close to $100 \%$ (sensitivity was $79.52 \%$ ). This means that $\sim 20 \%$ of the metastatic/recurrent tumours could not be classified using the integrated MR44 signature. This could indicate 
two possibilities: first, there are other types of tumour-specific changes that need to be

assayed and accounted for in order to derive a truly complete distant metastasis and recurrence-associated signature, and/or, second: the sample size of 83

metastatic/recurrent tumours was not sufficient (the predictive power of discovery was not optimum). The fact that the validation sensitivity of MR44 was $100 \%$ in an independent cohort does not deter from either of these conclusions as the number of tumours in the validation set was small $(n=18)$.

About half of the genes in the MR44 signature have been reported previously to be associated with prognosis, survival, recurrence and distant metastasis for various cancer types. For example, ITGA9 is reported as a tumour suppressor gene in non-small cell lung cancer (Pastuszak-Lewandoska et al, 2016) and is involved in cell migration and invasion in melanoma (Zhang et al, 2016). Additionally, epigenetic inactivation of ITGA9 is linked with its expression in nasopharyngeal and breast cancer (Mostovich et al, 2011; Nawaz et al, 2015). NRAS is linked with survival of patients with liver metastases in colorectal cancer (Vauthey et al, 2013) and acts as a prognostic factor in metastatic melanoma (Jakob et al, 2012). The chromosomal region containing DVL2 gene frequently undergoes LOH in patients with colorectal tumours (Kurashina et al, 2008). RPL26A1 expression was a prognostic marker in ER-positive breast tumours (Wang \& Zhang, 2007) and liver metastases in colorectal cancer (Nakamura \& Furukawa, 2003). MORF4L1 was found to be one of the candidate genes with copy number reductions in breast cancer (Chen et al, 2007). FGFR4 profile was observed to be prognostically relevant in squamous cell carcinoma of the mouth and oropharynx (Dutra et al, 2012), esophagus (Shim et al, 2016), and gastric cancer (Murase et al, 2014). ANXA11 expression is one of the prognostic markers in prostate cancer (Chandran et al, 2007; Tsai et al, 2013). ZBTB7A plays a role in suppressing tumour metastasis in gastric cancer (Shi et al, 2015). Loss of stromal CAV1 
expression predicts poor survival in colorectal cancer (Zhao et al, 2015). ARHGEF38

expression significantly differed between high and low recurrence-free survival groups in prostrate cancer (Tandefelt et al, 2013). OXCT1 was shown to act as an oncogene in human breast cancer cells (Martinez-Outschoorn et al, 2012). RICTOR regulates cancer cell metastases in breast cancer cell lines (Zhang et al, 2010). RPAP2 associates with breast cancer recurrence (Baker et al, 2014). mIR573 inhibits prostate cancer metastases (Wang et al, 2015). AIMP1 is down regulated in gastric and colorectal cancer (Kim et al, 2014). FAM134C is a cancer-relapse marker in breast and ovarian cancer (Guo, 2011). LIMS1 (PINCH signalling) has been linked to distant metastasis in pancreatic stromal cells (Scaife et al, 2010). EGLN1 (commonly referred to as PHD2) is an oxygen sensor that promotes breast cancer metastases (Kuchnio et al, 2015). RPLP2 is one of the 10-gene prognostic markers for gastric cancer (Zhang et al, 2011). TOLLIP was found to be significantly and differentially expressed in colorectal metastatic cells (Barderas et al, 2013). ZNF490 was found to be associated with breast cancer recurrence (Baker et al, 2013). MR44 contains genes implicated in MTOR, MAPK and PI3K-AKT signalling pathways via DVL2, FGF10, FGFR4, RICTOR, ITGA9, NRAS and CAV1 genes (Supplementary Table S4).

Although the discovery module of CAFE MOCHA uses 450k methylation array and assays being performed on the same platform so long as the user provides data in the same format for a matched normal. Future versions of CAFE MOCHA will incorporate cross-platform converter wherein the user can deposit data generated with multiple platforms and in multiple formats, thus making the tool truly platform agnostic. Additionally,

403 CAFE MOCHA has the ability to use an underlying tissue-specific normal database, 404 especially where expression and methylation data is not available, for un-matched normal 
bioRxiv preprint doi: https://doi.org/10.1101/105577; this version posted February 3, 2017. The copyright holder for this preprint (which was not certified by peer review) is the author/funder, who has granted bioRxiv a license to display the preprint in perpetuity. It is made available under aCC-BY 4.0 International license.

406

407

408

409

410

411

412

413

414

415

416

417

418

419

420

same assays and formats, used in the discovery module to minimize assay/platform

related errors.

\section{Funding}

Research presented in this article is funded by Department of Electronics and

Information Technology, Government of India (Ref No:18(4)/2010-E-Infra., 31-03-

2010) and Department of IT, BT and ST, Government of Karnataka, India (Ref

No:3451-00-090-2-22).

\section{References}

Agrawal N, Frederick MJ, Pickering CR, Bettegowda C, Chang K, Li RJ, Fakhry C, Xie TX, Zhang J, Wang J, Zhang N, El-Naggar AK, Jasser SA, Weinstein JN, Trevino L, Drummond JA, Muzny DM, Wu Y, Wood LD, Hruban RH, Westra WH, Koch WM, Califano JA, Gibbs RA, Sidransky D, Vogelstein B, Velculescu VE, Papadopoulos N, Wheeler DA, Kinzler KW, Myers JN (2011) Exome sequencing of head and neck squamous cell carcinoma reveals inactivating mutations in NOTCH1. Science 333(6046): 1154-7

Aryee MJ, Jaffe AE, Corrada-Bravo H, Ladd-Acosta C, Feinberg AP, Hansen KD, Irizarry RA (2014) Minfi: a flexible and comprehensive Bioconductor package for the analysis of Infinium DNA methylation microarrays. Bioinformatics 30(10): 1363-9

Baker JB, Sinicropi DV, Pelham RJ, Crager M, Collin F, Stephans JC, Liu M, Morlan J, Qu $\mathrm{K}$ (2013) Method of predicting breast cancer prognosis.

Baker JB, Sinicropi DV, Pelham RJ, Crager M, Collin F, Stephans JC, Liu M, Morlan J, Qu $\mathrm{K}$ (2014) Method of predicting breast cancer prognosis

Barderas R, Mendes M, Torres S, Bartolome RA, Lopez-Lucendo M, Villar-Vazquez R, Pelaez-Garcia A, Fuente E, Bonilla F, Casal Jl (2013) In-depth characterization of the secretome of colorectal cancer metastatic cells identifies key proteins in cell adhesion, migration, and invasion. Mol Cell Proteomics 12(6): 1602-20

Breiman L (2001) Random Forests. Vol. 45. The Netherlands: Kliwer Academic Publishers

Cancer Genome Atlas N (2015) Comprehensive genomic characterization of head and neck squamous cell carcinomas. Nature 517(7536): 576-82

Chandran UR, Ma C, Dhir R, Bisceglia M, Lyons-Weiler M, Liang W, Michalopoulos G, Becich M, Monzon FA (2007) Gene expression profiles of prostate cancer reveal involvement of multiple molecular pathways in the metastatic process. BMC Cancer 7: 64 
450 Chen W, Salto-Tellez M, Palanisamy N, Ganesan K, Hou Q, Tan LK, Sii LH, Ito K, Tan B, identified by integrative genomics. Genes, Chromosomes and Cancer 46(3): 288-301

Chou CH, Chang NW, Shrestha S, Hsu SD, Lin YL, Lee WH, Yang CD, Hong HC, Wei TY, Tu SJ, Tsai TR, Ho SY, Jian TY, Wu HY, Chen PR, Lin NC, Huang HT, Yang TL, Pai CY, Tai CS, Chen WL, Huang CY, Liu CC, Weng SL, Liao KW, Hsu WL, Huang HD (2016) miRTarBase 2016: updates to the experimentally validated miRNA-target interactions database. Nucleic acids research 44(D1): D239-47

Deng M, Bragelmann J, Schultze JL, Perner S (2016) Web-TCGA: an online platform for integrated analysis of molecular cancer data sets. BMC bioinformatics 17: 72

Dutra RL, de Carvalho MB, Dos Santos M, Mercante AM, Gazito D, de Cicco R, Group G, Tajara EH, Louro ID, da Silva AM (2012) FGFR4 profile as a prognostic marker in squamous cell carcinoma of the mouth and oropharynx. PLoS One 7(11): e50747

Editorial (2015) The future of cancer genomics. Nature medicine 21(2): 99

Ferlay J, Shin HR, Bray F, Forman D, Mathers C, Parkin DM (2010) Estimates of worldwide burden of cancer in 2008: GLOBOCAN 2008. International journal of cancer Journal international du cancer 127(12): 2893-917

Gao BJ. Hyper-rectangle-based discriminative data generalization and applications in data mining. Ph.D., Hyper-rectangle-based discriminative data generalization and applications in data mining. Ph.D. Thesis. Simon Fraser University, Canada, 2007

Gevaert O, Plevritis S (2013) Identifying master regulators of cancer and their downstream targets by integrating genomic and epigenomic features. Pacific Symposium on Biocomputing Pacific Symposium on Biocomputing: 123-34

Goh ST, Rubin C (2014) Box Drawings for Learning with Imbalanced Data aRxiv [statML]

Gundem G, Perez-Llamas C, Jene-Sanz A, Kedzierska A, Islam A, Deu-Pons J, Furney SJ, Lopez-Bigas N (2010) IntOGen: integration and data mining of multidimensional oncogenomic data. Nature methods 7(2): 92-3

Guo NL (2011) Gene signature for diagnosis and prognosis of breast cancer and ovarian cancer: Google Patents. US8030060

He Y, Zhang M, Ju Y, Yu Z, Lv D, Sun H, Yuan W, He F, Zhang J, Li H, Li J, Wang-Sattler R, Li Y, Zhang G, Xie L (2012) dbDEPC 2.0: updated database of differentially expressed proteins in human cancers. Nucleic acids research 40(Database issue): D964-71

Hsu SD, Lin FM, Wu WY, Liang C, Huang WC, Chan WL, Tsai WT, Chen GZ, Lee CJ, Chiu CM, Chien CH, Wu MC, Huang CY, Tsou AP, Huang HD (2011) miRTarBase: a database curates experimentally validated microRNA-target interactions. Nucleic acids research 39(Database issue): D163-9

Hsu SD, Tseng YT, Shrestha S, Lin YL, Khaleel A, Chou CH, Chu CF, Huang HY, Lin CM, Ho SY, Jian TY, Lin FM, Chang TH, Weng SL, Liao KW, Liao IE, Liu CC, Huang HD (2014) miRTarBase update 2014: an information resource for experimentally validated 
bioRxiv preprint doi: https://doi.org/10.1101/105577; this version posted February 3, 2017. The copyright holder for this preprint (which was not certified by peer review) is the author/funder, who has granted bioRxiv a license to display the preprint in perpetuity. It is made available under aCC-BY 4.0 International license.

502

503

504

505

506

507

508

509

510

511

512

513

514

515

516

517

518

519

520

521

522

523

524

525

526

527

528

529

530

531

532

533

534

535

536

537

538

539

540

541

542

543

544

545

546

547

548

549

550

551

552

553

miRNA-target interactions. Nucleic acids research 42(Database issue): D78-85

Huang WY, Hsu SD, Huang HY, Sun YM, Chou CH, Weng SL, Huang HD (2015) MethHC: a database of DNA methylation and gene expression in human cancer. Nucleic acids research 43(Database issue): D856-61

Hudson TJ, Anderson W, Artez A, Barker AD, Bell C, Bernabe RR, Bhan MK, Calvo F, Eerola I, Gerhard DS, Guttmacher A, Guyer M, Hemsley FM, Jennings JL, Kerr D, Klatt P, Kolar P, Kusada J, Lane DP, Laplace F, Youyong L, Nettekoven G, Ozenberger B, Peterson J, Rao TS, Remacle J, Schafer AJ, Shibata T, Stratton MR, Vockley JG, Watanabe K, Yang H, Yuen MM, Knoppers BM, Bobrow M, Cambon-Thomsen A, Dressler LG, Dyke SO, Joly Y, Kato K, Kennedy KL, Nicolas P, Parker MJ, Rial-Sebbag E, RomeoCasabona CM, Shaw KM, Wallace S, Wiesner GL, Zeps N, Lichter P, Biankin AV, Chabannon C, Chin L, Clement B, de Alava E, Degos F, Ferguson ML, Geary P, Hayes DN, Hudson TJ, Johns AL, Kasprzyk A, Nakagawa H, Penny R, Piris MA, Sarin R, Scarpa A, Shibata T, van de Vijver M, Futreal PA, Aburatani H, Bayes M, Botwell DD, Campbell PJ, Estivill X, Gerhard DS, Grimmond SM, Gut I, Hirst M, Lopez-Otin C, Majumder P, Marra M, McPherson JD, Nakagawa H, Ning Z, Puente XS, Ruan Y, Shibata T, Stratton MR, Stunnenberg HG, Swerdlow H, Velculescu VE, Wilson RK, Xue HH, Yang L, Spellman PT, Bader GD, Boutros PC, Campbell PJ, Flicek P, Getz G, Guigo R, Guo G, Haussler D, Heath S, Hubbard TJ, Jiang T, Jones SM, Li Q, Lopez-Bigas N, Luo R, Muthuswamy L, Ouellette BF, Pearson JV, Puente XS, Quesada V, Raphael BJ, Sander C, Shibata T, Speed TP, Stein LD, Stuart JM, Teague JW, Totoki Y, Tsunoda T, Valencia A, Wheeler DA, Wu H, Zhao S, Zhou G, Stein LD, Guigo R, Hubbard TJ, Joly Y, Jones SM, Kasprzyk A, Lathrop M, Lopez-Bigas N, Ouellette BF, Spellman PT, Teague JW, Thomas G, Valencia A, Yoshida T, Kennedy KL, Axton M, Dyke SO, Futreal PA, Gerhard DS, Gunter C, Guyer M, Hudson TJ, McPherson JD, Miller LJ, Ozenberger B, Shaw KM, Kasprzyk A, Stein LD, Zhang J, Haider SA, Wang J, Yung CK, Cros A, Liang Y, Gnaneshan S, Guberman J, Hsu J, Bobrow M, Chalmers DR, Hasel KW, Joly Y, Kaan TS, Kennedy KL, Knoppers BM, Lowrance WW, Masui T, Nicolas P, Rial-Sebbag E, Rodriguez LL, Vergely C, Yoshida T, Grimmond SM, Biankin AV, Bowtell DD, Cloonan N, deFazio A, Eshleman JR, Etemadmoghadam D, Gardiner BB, Kench JG, Scarpa A, Sutherland RL, Tempero MA, Waddell NJ, Wilson PJ, McPherson JD, Gallinger S, Tsao MS, Shaw PA, Petersen GM, Mukhopadhyay D, Chin L, DePinho RA, Thayer S, Muthuswamy L, Shazand K, Beck T, Sam M, Timms L, Ballin V, Lu Y, Ji J, Zhang X, Chen F, Hu X, Zhou G, Yang Q, Tian G, Zhang L, Xing X, Li X, Zhu Z, Yu Y, Yu J, Yang H, Lathrop M, Tost J, Brennan P, Holcatova I, Zaridze D, Brazma A, Egevard L, Prokhortchouk E, Banks RE, Uhlen M, Cambon-Thomsen A, Viksna J, Ponten F, Skryabin K, Stratton MR, Futreal PA, Birney E, Borg A, Borresen-Dale AL, Caldas C, Foekens JA, Martin S, Reis-Filho JS, Richardson AL, Sotiriou C, Stunnenberg HG, Thoms G, van de Vijver M, van't Veer L, Calvo F, Birnbaum D, Blanche H, Boucher P, Boyault S, Chabannon C, Gut I, Masson-Jacquemier JD, Lathrop M, Pauporte I, Pivot X, Vincent-Salomon A, Tabone E, Theillet C, Thomas G, Tost J, Treilleux I, Calvo F, Bioulac-Sage P, Clement B, Decaens T, Degos F, Franco D, Gut I, Gut M, Heath S, Lathrop M, Samuel D, Thomas G, Zucman-Rossi J, Lichter P, Eils R, Brors B, Korbel JO, Korshunov A, Landgraf P, Lehrach H, Pfister S, Radlwimmer B, Reifenberger G, Taylor MD, von Kalle C, Majumder PP, Sarin R, Rao TS, Bhan MK, Scarpa A, Pederzoli P, Lawlor RA, Delledonne M, Bardelli A, Biankin AV, Grimmond SM, Gress T, Klimstra D, Zamboni G, Shibata T, Nakamura Y, Nakagawa H, Kusada J, Tsunoda T, Miyano S, Aburatani H, Kato K, Fujimoto A, Yoshida T, Campo E, Lopez-Otin C, Estivill X, Guigo R, de Sanjose S, Piris MA, Montserrat E, Gonzalez-Diaz M, Puente XS, Jares P, Valencia A, Himmelbauer H, Quesada V, Bea S, Stratton MR, Futreal PA, Campbell PJ, Vincent-Salomon A, Richardson AL, Reis-Filho JS, van de Vijver M, Thomas 
G, Masson-Jacquemier JD, Aparicio S, Borg A, Borresen-Dale AL, Caldas C, Foekens JA, Stunnenberg HG, van't Veer L, Easton DF, Spellman PT, Martin S, Barker AD, Chin L, Collins FS, Compton CC, Ferguson ML, Gerhard DS, Getz G, Gunter C, Guttmacher A, Guyer M, Hayes DN, Lander ES, Ozenberger B, Penny R, Peterson J, Sander C, Shaw KM, Speed TP, Spellman PT, Vockley JG, Wheeler DA, Wilson RK, Hudson TJ, Chin L, Knoppers BM, Lander ES, Lichter P, Stein LD, Stratton MR, Anderson W, Barker AD, Bell C, Bobrow M, Burke W, Collins FS, Compton CC, DePinho RA, Easton DF, Futreal PA, Gerhard DS, Green AR, Guyer M, Hamilton SR, Hubbard TJ, Kallioniemi OP, Kennedy KL, Ley TJ, Liu ET, Lu Y, Majumder P, Marra M, Ozenberger B, Peterson J, Schafer AJ, Spellman PT, Stunnenberg HG, Wainwright BJ, Wilson RK, Yang H (2010) International network of cancer genome projects. Nature 464(7291): 993-8

India Project Team of the International Cancer Genome C (2013) Mutational landscape of gingivo-buccal oral squamous cell carcinoma reveals new recurrently-mutated genes and molecular subgroups. Nature communications 4: 2873

Iwamoto T, Pusztai L (2010) Predicting prognosis of breast cancer with gene signatures: are we lost in a sea of data? Genome medicine 2(11): 81

Jakob JA, Bassett RL, Jr., Ng CS, Curry JL, Joseph RW, Alvarado GC, Rohlfs ML, Richard J, Gershenwald JE, Kim KB, Lazar AJ, Hwu P, Davies MA (2012) NRAS mutation status is an independent prognostic factor in metastatic melanoma. Cancer 118(16): 4014-23

Kanehisa M, Goto S, Kawashima S, Nakaya A (2002) The KEGG databases at GenomeNet. Nucleic acids research 30(1): 42-6

Kanehisa M, Goto S, Kawashima S, Okuno Y, Hattori M (2004) The KEGG resource for deciphering the genome. Nucleic acids research 32(Database issue): D277-80

Kanehisa M, Goto S, Sato Y, Furumichi M, Tanabe M (2012) KEGG for integration and interpretation of large-scale molecular data sets. Nucleic acids research 40(1): D109-14

Kim MS, Kim S, Myung H (2014) Degradation of AIMP1/p43 induced by hepatitis C virus E2 leads to upregulation of TGF-beta signaling and increase in surface expression of gp96. PLoS One 9(5): e96302

kirmse M, Petersohn U (2011) Large margin rectangle learning an alternative way to learn interpretable and representative models. In International Conference of Soft Computing and Pattern Recognition (SoCPaR) pp 161-166: IEEE

Krishnan N, Gupta S, Palve V, Varghese L, Pattnaik S, Jain P, Khyriem C, Hariharan A, Dhas K, Nair J, Pareek M, Prasad V, Siddappa G, Suresh A, Kekatpure V, Kuriakose M, Panda B (2015) Integrated analysis of oral tongue squamous cell carcinoma identifies key variants and pathways linked to risk habits, HPV, clinical parameters and tumor recurrence. F1000Res 4: 1215

Krishnan NM, Dhas K, Nair J, Palve V, Bagwan J, Siddappa G, Suresh A, Kekatpure VD, Kuriakose MA, Panda B (2016) A Minimal DNA Methylation Signature in Oral Tongue Squamous Cell Carcinoma Links Altered Methylation with Tumor Attributes. Mol Cancer Res 14(9): 805-19

Kuchnio A, Dewerchin M, Carmeliet P (2015) The PHD2 oxygen sensor paves the way to 
606

607

608

609

610

611

612

613

614

615

616

617

618

619

620

621

622

623

624

625

626

627

628

629

630

631

632

633

634

635

636

637

638

639

640

metastasis. Oncotarget 6(34): 35149-50

Kurashina K, Yamashita Y, Ueno T, Koinuma K, Ohashi J, Horie H, Miyakura Y, Hamada T, Haruta H, Hatanaka H, Soda M, Choi YL, Takada S, Yasuda Y, Nagai H, Mano H (2008) Chromosome copy number analysis in screening for prognosis-related genomic regions in colorectal carcinoma. Cancer Sci 99(9): 1835-40

Li B, Dewey CN (2011) RSEM: accurate transcript quantification from RNA-Seq data with or without a reference genome. BMC bioinformatics 12: 323

Martinez-Outschoorn UE, Lin Z, Whitaker-Menezes D, Howell A, Sotgia F, Lisanti MP (2012) Ketone body utilization drives tumor growth and metastasis. Cell Cycle 11(21): 3964-71

Mishra A, Meherotra R (2014) Head and neck cancer: global burden and regional trends in India. Asian Pacific journal of cancer prevention : APJCP 15(2): 537-50

Mosca E, Alfieri R, Merelli I, Viti F, Calabria A, Milanesi L (2010) A multilevel data integration resource for breast cancer study. BMC systems biology 4: 76

Mostovich LA, Prudnikova TY, Kondratov AG, Loginova D, Vavilov PV, Rykova VI, Sidorov SV, Pavlova TV, Kashuba VI, Zabarovsky ER, Grigorieva EV (2011) Integrin alpha9 (ITGA9) expression and epigenetic silencing in human breast tumors. Cell Adh Migr 5(5): 395-401

Murase H, Inokuchi M, Takagi Y, Kato K, Kojima K, Sugihara K (2014) Prognostic significance of the co-overexpression of fibroblast growth factor receptors 1, 2 and 4 in gastric cancer. Mol Clin Oncol 2(4): 509-517

Nakamura Y, Furukawa Y (2003) Method for treating or preventing metastasis of colorectal cancers: Google Patents. US20060111314

Nawaz I, Hu LF, Du ZM, Moumad K, Ignatyev I, Pavlova TV, Kashuba V, Almgren M, Zabarovsky ER, Ernberg I (2015) Integrin alpha9 gene promoter is hypermethylated and downregulated in nasopharyngeal carcinoma. Oncotarget 6(31): 31493-507

Netanely D, Avraham A, Ben-Baruch A, Evron E, Shamir R (2016) Expression and methylation patterns partition luminal-A breast tumors into distinct prognostic subgroups. Breast cancer research : BCR 18(1): 74

Pastuszak-Lewandoska D, Kordiak J, Antczak A, Migdalska-Sek M, Czarnecka KH, Gorski P, Nawrot E, Kiszalkiewicz JM, Domanska-Senderowska D, Brzezianska-Lasota E (2016) Expression level and methylation status of three tumor suppressor genes, DLEC1, ITGA9 and MLH1, in non-small cell lung cancer. Med Oncol 33(7): 75

Pickering CR, Zhang J, Yoo SY, Bengtsson L, Moorthy S, Neskey DM, Zhao M, Ortega Alves MV, Chang K, Drummond J, Cortez E, Xie TX, Zhang D, Chung W, Issa JP, Zweidler-McKay PA, Wu X, El-Naggar AK, Weinstein JN, Wang J, Muzny DM, Gibbs RA, Wheeler DA, Myers JN, Frederick MJ (2013) Integrative genomic characterization of oral squamous cell carcinoma identifies frequent somatic drivers. Cancer discovery 3(7): 77081 
658

659

660

661

662

663

664

665

666

667

668

669

670

671

672

673

674

675

676

677

678

679

680

681

682

683

684

685

686

687

688

689

690

691

692

693

694

695

696

697

698

699

700

701

702

703

704

705

706

707

708

709

Pidsley R, CC YW, Volta M, Lunnon K, Mill J, Schalkwyk LC (2013) A data-driven approach to preprocessing Illumina 450K methylation array data. BMC genomics 14: 293

Richards JA (2013) Remote Sensing Digital Image Analysis: An Introduction: Springer Science \& Business Media

Ridge JA, Mehra R, Lango MN, Galloway T (2016) Head and neck tumors. In Cancer management: a multidisciplinary approach., Haller DG, Wagman LD, Camphausen KA, Hoskins WJ (eds), Chapter 2.

Scaife CL, Shea J, Emerson L, Boucher K, Firpo MA, Beckerle MC, Mulvihill SJ (2010) Prognostic significance of PINCH signalling in human pancreatic ductal adenocarcinoma. HPB (Oxford) 12(5): 352-8

Shi DB, Wang YW, Xing AY, Gao JW, Zhang H, Guo XY, Gao P (2015) C/EBPalphainduced miR-100 expression suppresses tumor metastasis and growth by targeting ZBTB7A in gastric cancer. Cancer Lett 369(2): 376-85

Shim HJ, Shin MH, Kim HN, Kim JH, Hwang JE, Bae WK, Chung IJ, Cho SH (2016) The Prognostic Significance of FGFR4 Gly388 Polymorphism in Esophageal Squamous Cell Carcinoma after Concurrent Chemoradiotherapy. Cancer Res Treat 48(1): 71-9

Tandefelt DG, Boormans JL, van der Korput HA, Jenster GW, Trapman J (2013) A 36gene signature predicts clinical progression in a subgroup of ERG-positive prostate cancers. European urology 64(6): 941-950

Thingholm LB, Andersen L, Makalic E, Southey MC, Thomassen M, Hansen LL (2016) Strategies for Integrated Analysis of Genetic, Epigenetic, and Gene Expression Variation in Cancer: Addressing the Challenges. Frontiers in genetics 7: 2

Tsai K-CK, Chi-Rong L, SU J-MJ (2013) Molecular markers for prognostically predicting prostate cancer, method and kit thereof: Google Patents. US20130331281

Uhlen M, Fagerberg L, Hallstrom BM, Lindskog C, Oksvold P, Mardinoglu A, Sivertsson A, Kampf C, Sjostedt E, Asplund A, Olsson I, Edlund K, Lundberg E, Navani S, Szigyarto CA, Odeberg J, Djureinovic D, Takanen JO, Hober S, Alm T, Edqvist PH, Berling H, Tegel H, Mulder J, Rockberg J, Nilsson P, Schwenk JM, Hamsten M, von Feilitzen K, Forsberg M, Persson L, Johansson F, Zwahlen M, von Heijne G, Nielsen J, Ponten F (2015)

Proteomics. Tissue-based map of the human proteome. Science 347(6220): 1260419

Vauthey JN, Zimmitti G, Kopetz SE, Shindoh J, Chen SS, Andreou A, Curley SA, Aloia TA, Maru DM (2013) RAS mutation status predicts survival and patterns of recurrence in patients undergoing hepatectomy for colorectal liver metastases. Ann Surg 258(4): 61926; discussion 626-7

Wang L, Song G, Tan W, Qi M, Zhang L, Chan J, Yu J, Han J, Han B (2015) MiR-573 inhibits prostate cancer metastasis by regulating epithelial-mesenchymal transition. Oncotarget 6(34): 35978-90

Wang Y, Zhang Y (2007) Methods of predicting distant metastasis of lymph node-negative primary breast cancer using biological pathway gene expression analysis: Google Patents. US20080182246 
Weinstein JN, Collisson EA, Mills GB, Shaw KR, Ozenberger BA, Ellrott K, Shmulevich I, Sander C, Stuart JM (2013) The Cancer Genome Atlas Pan-Cancer analysis project.

Nature genetics 45(10): 1113-20

Zhang F, Zhang X, Li M, Chen P, Zhang B, Guo H, Cao W, Wei X, Cao X, Hao X, Zhang N (2010) mTOR complex component Rictor interacts with PKCzeta and regulates cancer cell metastasis. Cancer Res 70(22): 9360-70

Zhang J, Na S, Liu C, Pan S, Cai J, Qiu J (2016) MicroRNA-125b suppresses the epithelial-mesenchymal transition and cell invasion by targeting ITGA9 in melanoma. Tumour Biol 37(5): 5941-9

Zhang YZ, Zhang LH, Gao Y, Li CH, Jia SQ, Liu N, Cheng F, Niu DY, Cho WC, Ji JF, Zeng CQ (2011) Discovery and validation of prognostic markers in gastric cancer by genome-wide expression profiling. World J Gastroenterol 17(13): 1710-7

Zhao Z, Han FH, Yang SB, Hua LX, Wu JH, Zhan WH (2015) Loss of stromal caveolin-1 expression in colorectal cancer predicts poor survival. World J Gastroenterol 21(4): 1140-7 
bioRxiv preprint doi: https://doi.org/10.1101/105577; this version posted February 3, 2017. The copyright holder for this preprint (which was not certified by peer review) is the author/funder, who has granted bioRxiv a license to display the preprint in perpetuity. It is made available under aCC-BY 4.0 International license.

734 Table 1: Sample cohorts used in the discovery and validation modules of CAFE MOCHA

735 for discovering metastatic/recurrent signature MR44 in head and neck squamous cell 736 carcinoma (HNSCC).

\begin{tabular}{|c|c|c|c|}
\hline \multicolumn{4}{|c|}{ Discovery } \\
\hline \multirow[t]{4}{*}{ Stage 1} & Source & \multicolumn{2}{|c|}{$\begin{array}{l}\text { TCGA HNSCC (http://tinyurl.com/jhmg9b9), } \\
\text { data available on all the } 4 \text { event types (mut, } \\
\text { CNV, exp, meth) on tumor:matched normal } \\
\text { samples. }\end{array}$} \\
\hline & Total Number of tumors & \multicolumn{2}{|l|}{434} \\
\hline & Metastatic/Recurrent & \multicolumn{2}{|l|}{83} \\
\hline & $\begin{array}{l}\text { Tumors without } \\
\text { metastasis/recurrence }\end{array}$ & \multicolumn{2}{|l|}{351} \\
\hline \multirow[t]{6}{*}{ Stage 2} & Source & \multicolumn{2}{|c|}{$\begin{array}{l}\text { Data on at least one event type available from } \\
\text { the same matched tumor:normal sample. }\end{array}$} \\
\hline & & TCGA HNSCC & $\begin{array}{l}\text { OTSCC (Krishnan et } \\
\text { al, 2015; Krishnan et al, } \\
\text { 2016) }\end{array}$ \\
\hline & Total number of tumors & 42 & 37 \\
\hline & Metastatic/Recurrent & 4 & 15 \\
\hline & $\begin{array}{l}\text { Tumors without } \\
\text { metastasis/recurrence }\end{array}$ & 38 & 22 \\
\hline & Adjacent normal tissue & 0 & 37 \\
\hline \multicolumn{4}{|c|}{ Validation } \\
\hline & Source & \multicolumn{2}{|c|}{$\begin{array}{l}\text { OTSCC (Krishnan et al, 2015; Krishnan et al, } \\
\text { 2016) (matched tumor:normal data on all } \\
\text { event types from the same tumors) }\end{array}$} \\
\hline & Total number of tumors & \multicolumn{2}{|l|}{18} \\
\hline & Metastatic/Recurrent & \multicolumn{2}{|l|}{3} \\
\hline & $\begin{array}{l}\text { Tumors without } \\
\text { metastasis/recurrence }\end{array}$ & \multicolumn{2}{|l|}{15} \\
\hline & Adjacent normal tissue & \multicolumn{2}{|l|}{18} \\
\hline
\end{tabular}




\section{Figure Legends}

744 Figure 1: CAFE MOCHA application workflow and graphical-user-interface. A. Discovery,

745 and Prediction modules. B. Integrated analyses.

747 Figure 2: Complete specificity margin based clustering (CSMBC) algorithm for quantitative and coupled events.

749 Clustering of quantitative and coupled events, for three clinical sub-categories $i, j$ and $k$ is methylation to clinical sub-categories $i, j$ and $k$ (shades of blue). Boundaries are determined in a supervised manner, as the minimum and maximum limits of the quantitative ranges for each clinical sub-category. Overlaps are estimated as per the 754 equations illustrated in the figure. Samples whose expression or methylation values lie outside the regions of overlap, $100 \%$ specific to a clinical sub-category are factored in towards the sensitivity of that gene for that clinical sub-category. Panel B: clinical linking of coupled events such as fmeth (shades of orange) resulting from a combination of two quantitative events (expr - shades of blue and meth - shades of purple). The expression event is mapped along the first dimension and the methylation event is mapped along the second, and the samples, which observe both, expression and methylation, boundaries, contribute to the sensitivity of that fmeth event for that clinical sub-category. Panel C: clinical linking of coupled events such as fcnv (shades of dark orange) resulting from a 763 combination of one quantitative event (expression - shades of blue) and another 764 categorical event (CNV - shades of green). Here, the fcnv event linked to a clinical subcategory is determined by both, expression boundaries for a clinical sub-group, and presence of CNVs for the same samples in that clinical sub-group. 
768 Figure 3: Discovery heatmap (A) and per-event validation sensitivity (B) of 44-gene

769 integrated signature (MR44) for distant metastasis and recurrence in the HNSCC

770 discovery set.

771 An integrated signature associated with metastasis/recurrence was derived from

772 combining six event types (mut: red, fmeth: dark orange, fcnv: orange, cnv: green, expr:

773 blue and meth: purple). Cumulative sample frequency (\%) for individual event types is

774 represented as a histogram. The per-event validation sensitivity is represented as bubbles,

775 where the bubble size is proportional to the sample frequency of that event in the

776 validation cohort.

778 Figure 4: Comparison of detection sensitivities and total numbers of events required

779 achieving the sensitivity in one (A), two (B), three (C), four (D), five (E) and all six (F) event 780 types. Utilizing all the six events $(F)$ shows the power of integration both on sensitivity of

781 detection (black dot) and the number of events (colored bars for all the six individual event 782 types) required to attain the sensitivity.

\section{Supplementary Data Legend}

785 Supplementary Table S1: TCGA HNSCC tumor samples $(n=434)$ and clinical attributes used for the discovery of MR44.

788 Supplementary Table S2: Oral tongue squamous cell carcinoma (OTSCC) and TCGA 789 HNSCC samples lacking cross-platform overlap, and their clinical attributes used for 790 confirmation of MR44 discovery.

792 Supplementary Table S3: Oral tongue squamous cell carcinoma (OTSCC) $(n=18)$ with all 
793 four events assayed within the same tumor, and their clinical attributes used for the

794 validation.

795 Supplementary Table S4: Discovered events associated with Metastases and Recurrence 796 in HNSCC, pathways mapped, confirmation and validation status.

797

798 Supplementary Figure S1: Events associated with distant metastasis and recurrence in 799 HNSCC with single-event or multi-event signatures, selected in individual event type (mut: 800 red, fmeth: d orange, fcnv: orange, cnv: green, expr: blue and meth: purple) and various 801 integrated analyses, combining different numbers of event types. 
bioRxiv preprint doi: https://doi.org/10.1101/105577; this version posted February 3, 2017. The copyright holder for this preprint (which was not certifiêd by peer review) is the author/funder, who has granted bioRxiv a license to display the preprint in perpetuity. It is made

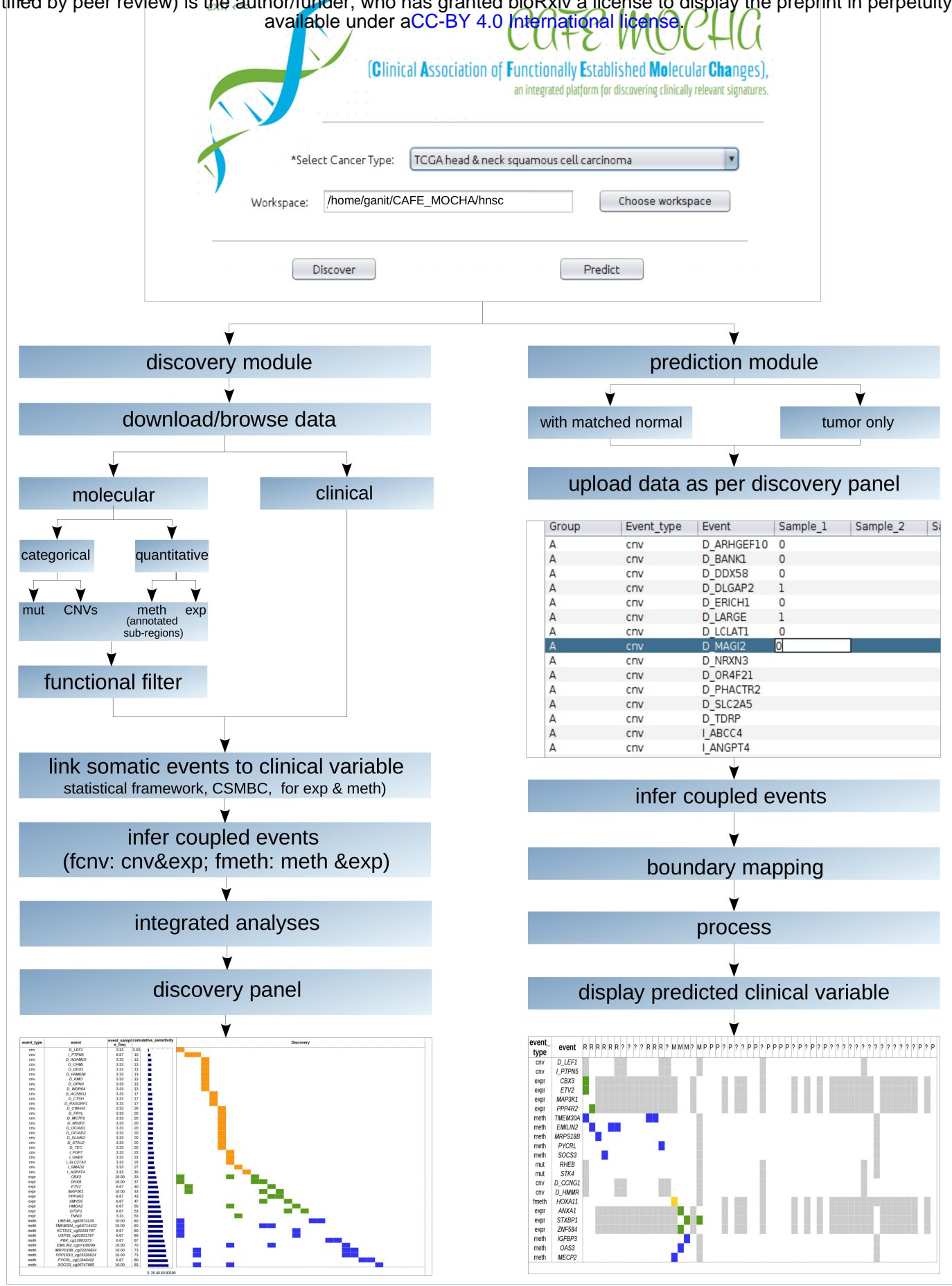

B
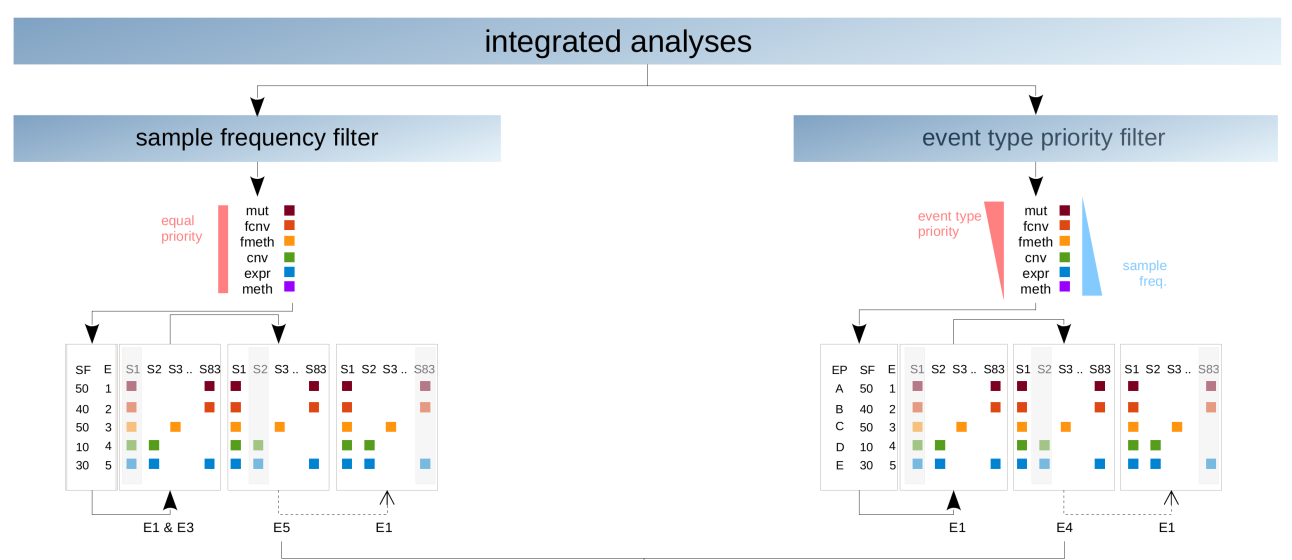

remove duplicates \& combine

E1, E3, E5 \& E4

$\downarrow$

removal of false positives

minimal signature

discovery panel 
bioRxiv preprint doi: https://doi.org/10.1101/105577; this version posted February 3, 2017. The copyright holder for this preprint (which was not certifed by peer review) is the author/funder, who has granted bioRxiv a license to display the preprint in perpetuity. It is made

exp/meth profile for a gene for clin vars $i, j, k$ - avâlable under ace-B 4.0 International license.

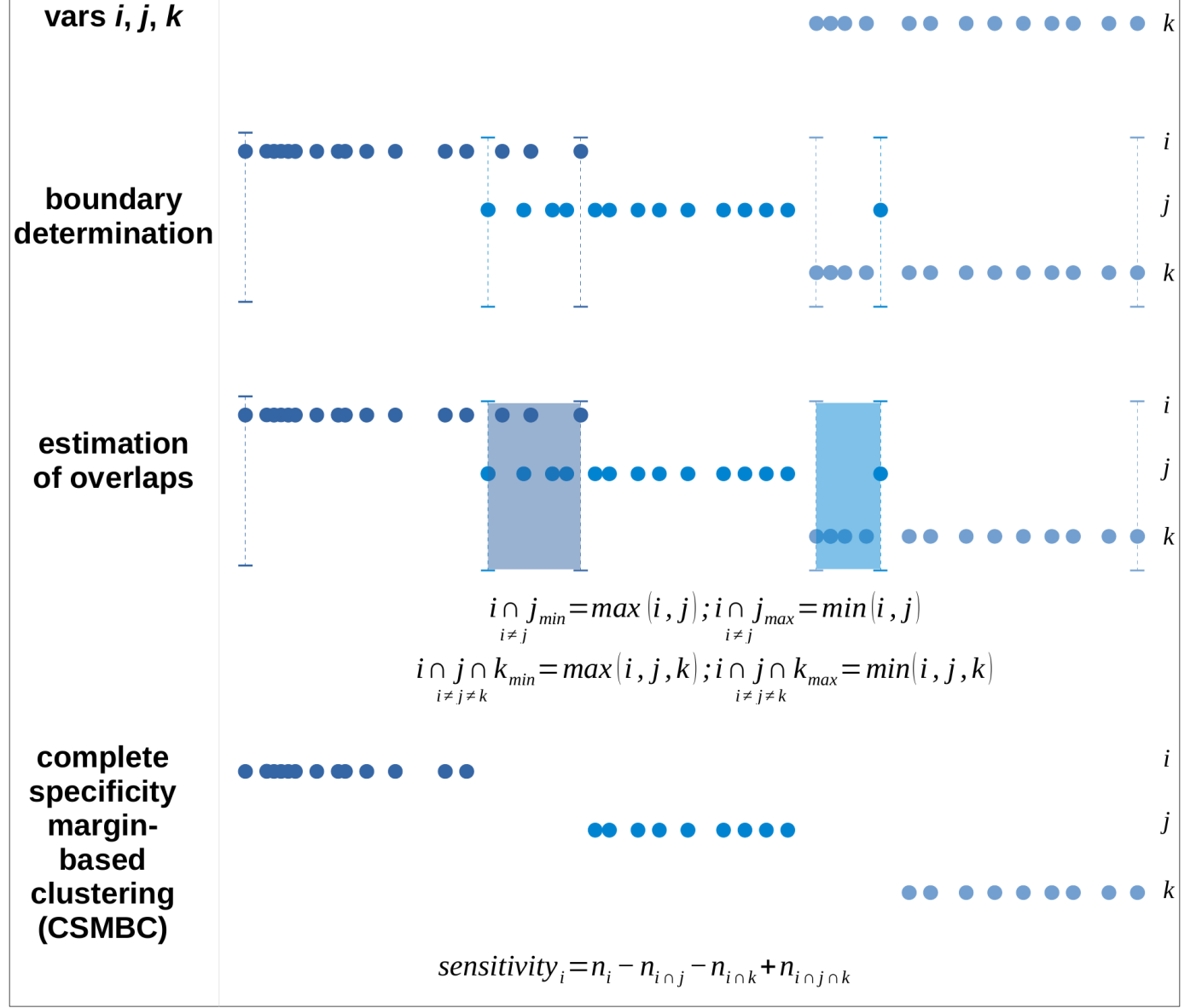

B

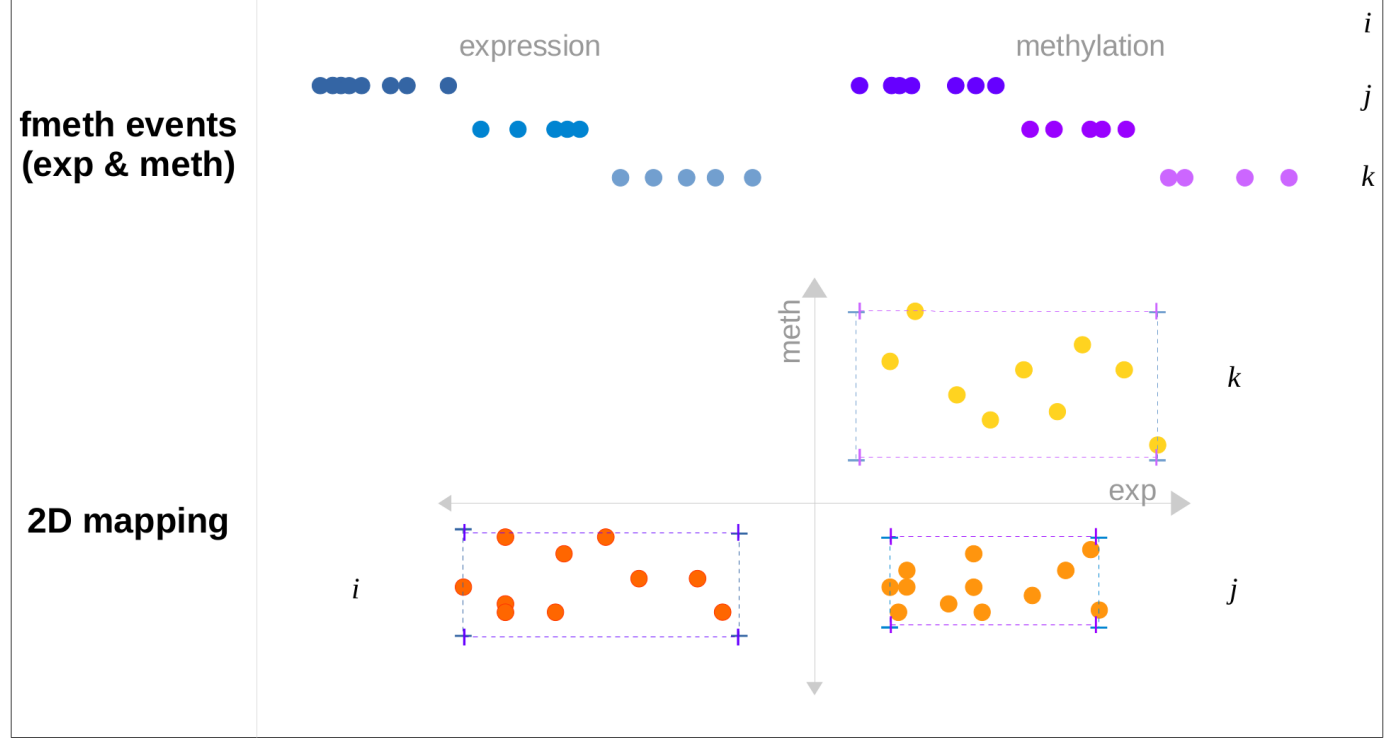

C

fcnv events

(exp \& cnv)
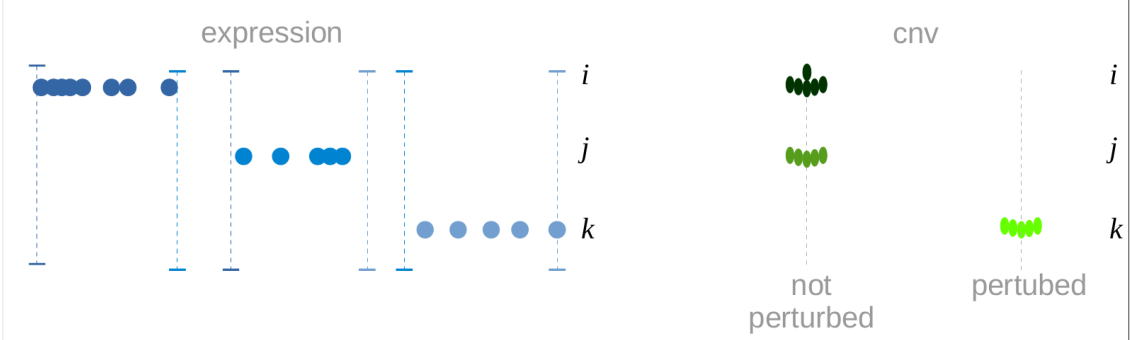

2D mapping 


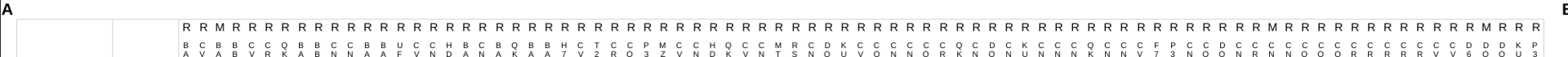

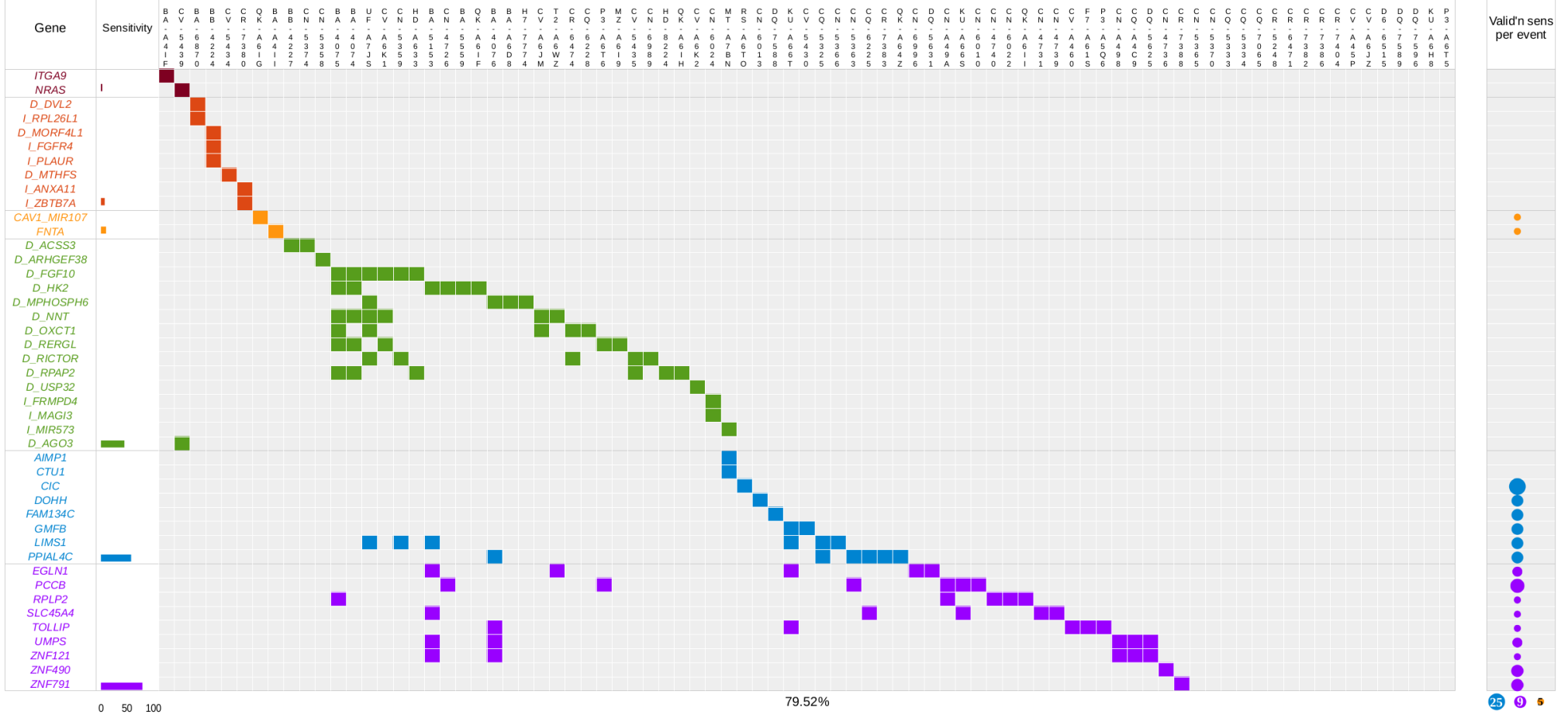




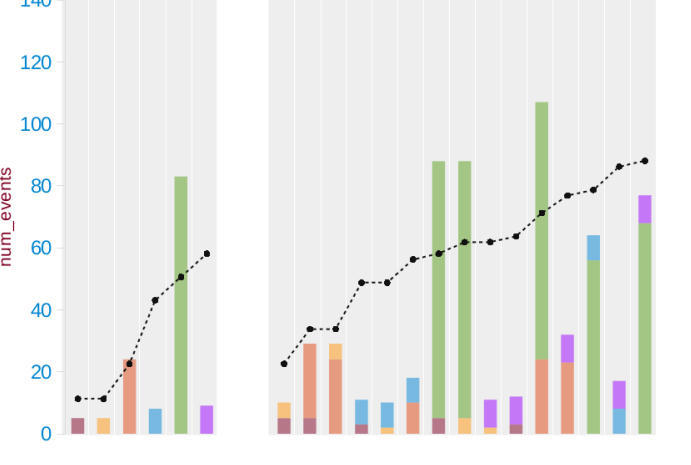




\section{"нm 1}

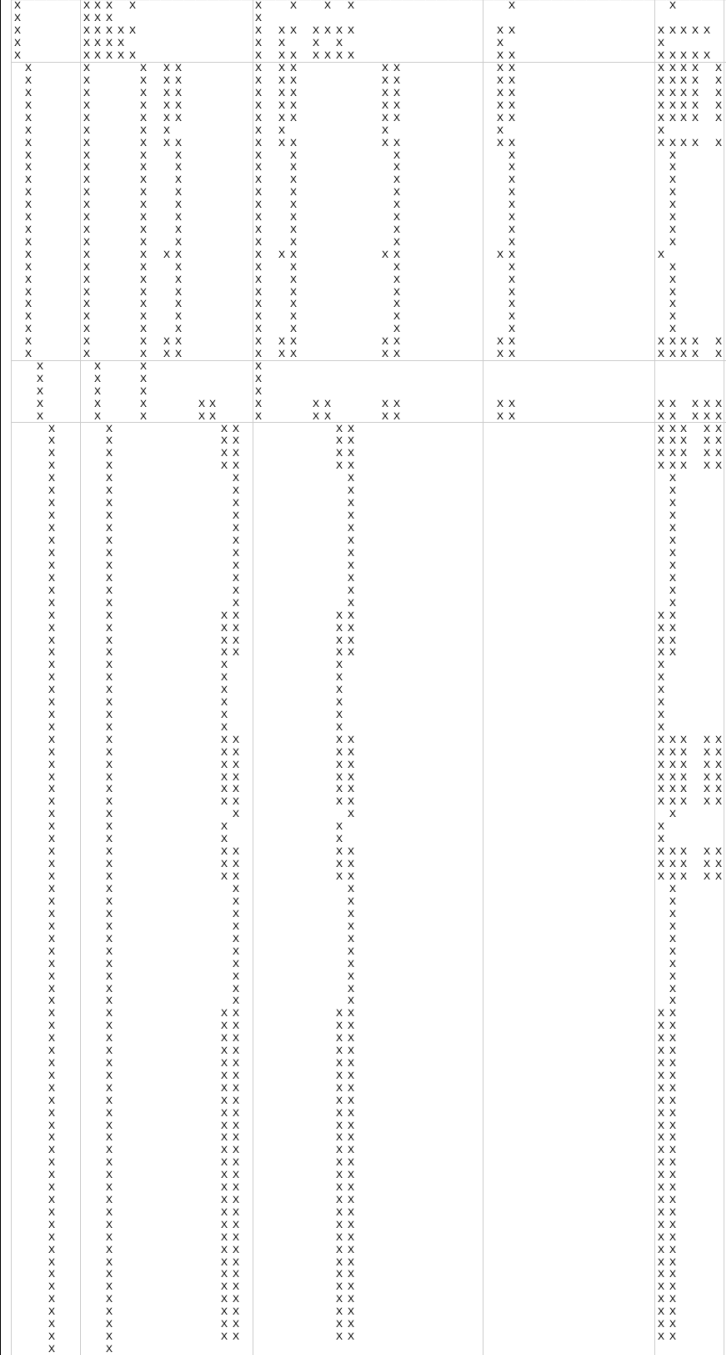

$x \times$
$x \times$
$x$
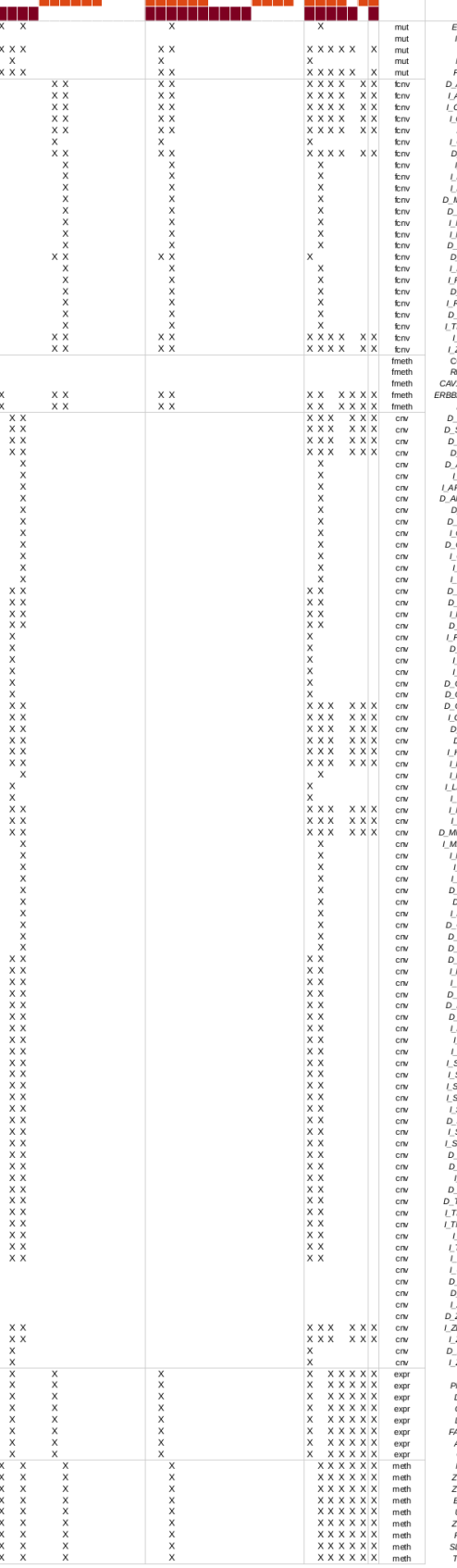

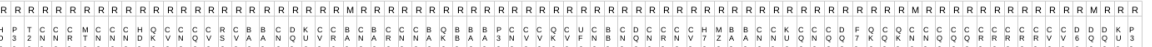

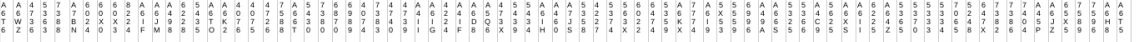

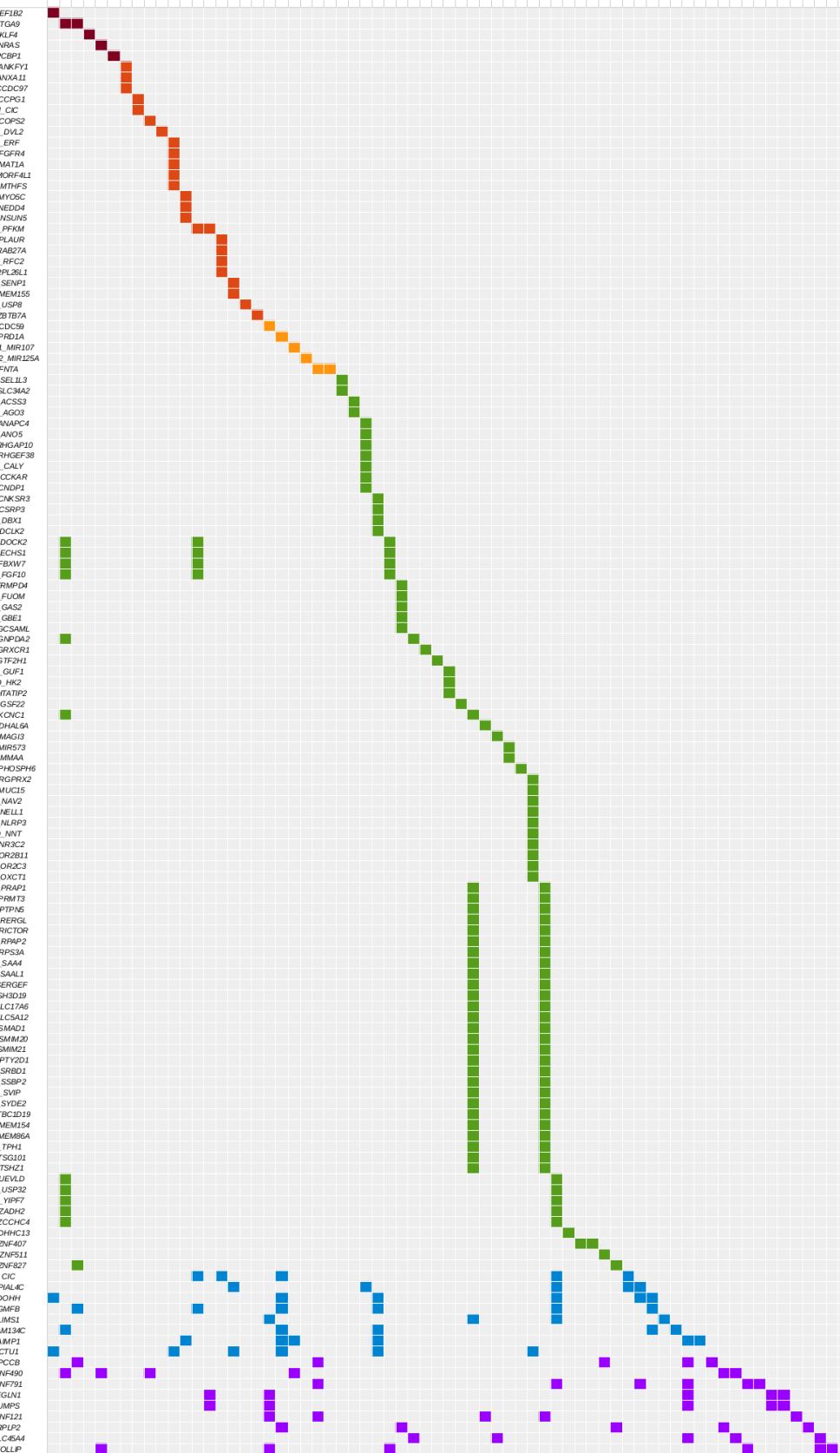


Supplementary Table S1: TCGA HNSCC samples and clinical attributes used for discovery.

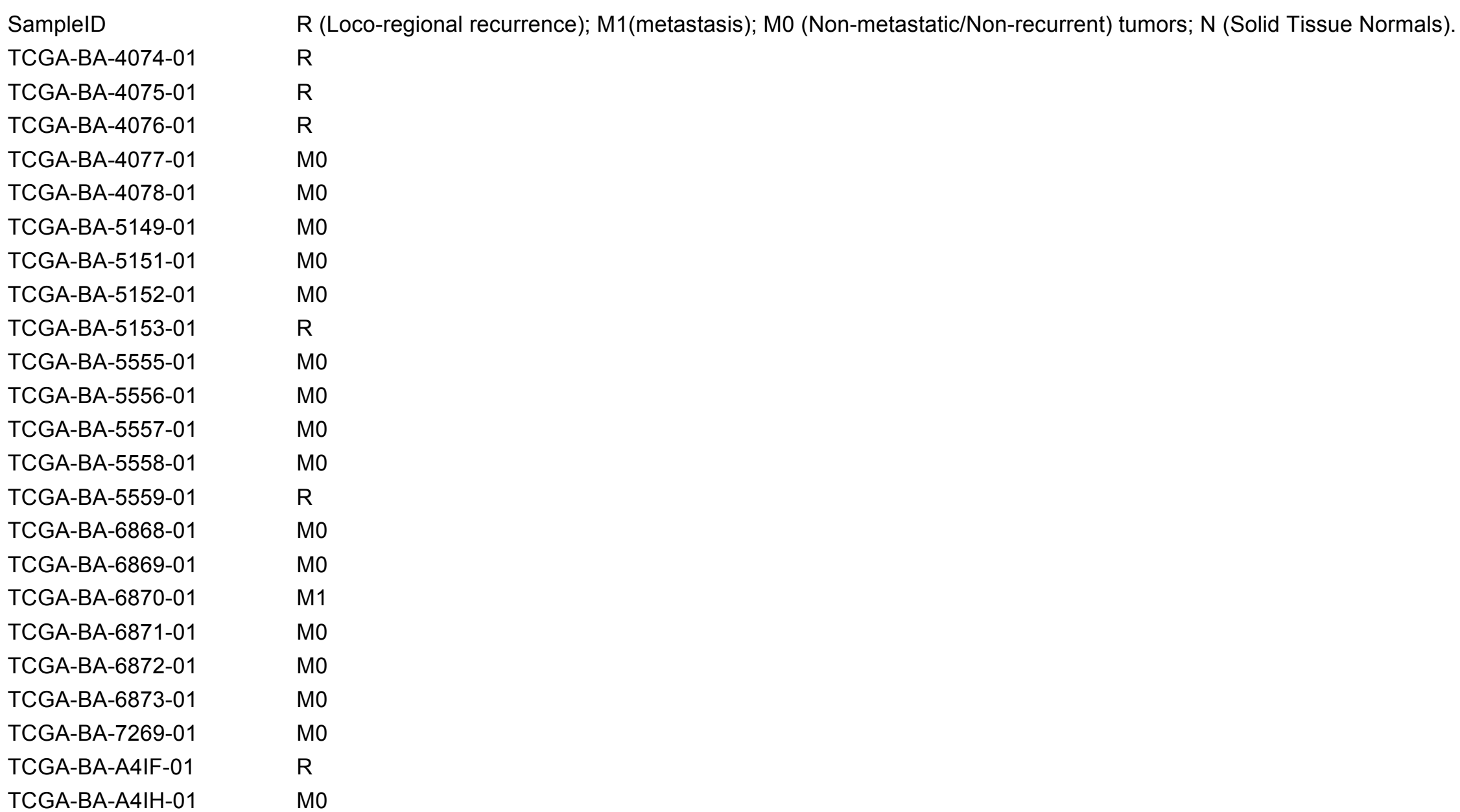




$\begin{array}{ll}\text { TCGA-BA-A4II-01 } & \text { R } \\ \text { TCGA-BA-A6D8-01 } & \text { R } \\ \text { TCGA-BA-A6DA-01 } & \text { M0 } \\ \text { TCGA-BA-A6DB-01 } & \text { M0 } \\ \text { TCGA-BA-A6DD-01 } & \text { M0 } \\ \text { TCGA-BA-A6DE-01 } & \text { M0 } \\ \text { TCGA-BA-A6DI-01 } & \text { M0 } \\ \text { TCGA-BA-A6DJ-01 } & \text { M0 } \\ \text { TCGA-BA-A6DL-01 } & \text { M0 } \\ \text { TCGA-BB-4217-01 } & \text { M0 } \\ \text { TCGA-BB-4223-01 } & \text { M0 } \\ \text { TCGA-BB-4224-01 } & \mathrm{R} \\ \text { TCGA-BB-4225-01 } & \text { M0 } \\ \text { TCGA-BB-4227-01 } & \mathrm{R} \\ \text { TCGA-BB-4228-01 } & \text { M0 } \\ \text { TCGA-BB-7861-01 } & \text { M0 } \\ \text { TCGA-BB-7862-01 } & \text { M0 } \\ \text { TCGA-BB-7863-01 } & \text { M0 } \\ \text { TCGA-BB-7864-01 } & \text { M0 } \\ \text { TCGA-BB-7870-01 } & \text { M0 } \\ \text { TCGA-BB-7871-01 } & \text { M0 } \\ \text { TCGA-BB-7872-01 } & \text { M0 } \\ \text { TCGA-C9-A47Z-01 } & \text { M0 } \\ \text { TCGA-C9-A480-01 } & \text { M0 } \\ \text { TCGA-CN-4723-01 } & \text { M0 } \\ \text { TCGA-CN-4725-01 } & \text { M0 } \\ \text { TCGA-CN-4726-01 } & \mathrm{R}\end{array}$




$\begin{array}{ll}\text { TCGA-CN-4727-01 } & \text { M0 } \\ \text { TCGA-CN-4728-01 } & \text { M0 } \\ \text { TCGA-CN-4729-01 } & \mathrm{M} 0 \\ \text { TCGA-CN-4730-01 } & \mathrm{M} 0 \\ \text { TCGA-CN-4731-01 } & \mathrm{R} \\ \text { TCGA-CN-4733-01 } & \mathrm{M} 0 \\ \text { TCGA-CN-4735-01 } & \mathrm{M} 0 \\ \text { TCGA-CN-4736-01 } & \mathrm{R} \\ \text { TCGA-CN-4737-01 } & \mathrm{M} 0 \\ \text { TCGA-CN-4738-01 } & \mathrm{M} 0 \\ \text { TCGA-CN-4739-01 } & \mathrm{R} \\ \text { TCGA-CN-4740-01 } & \mathrm{R} \\ \text { TCGA-CN-4741-01 } & \mathrm{M} 0 \\ \text { TCGA-CN-4742-01 } & \mathrm{M} 0 \\ \text { TCGA-CN-5355-01 } & \mathrm{M} 0 \\ \text { TCGA-CN-5356-01 } & \mathrm{M} 0 \\ \text { TCGA-CN-5358-01 } & \mathrm{R} \\ \text { TCGA-CN-5359-01 } & \mathrm{R} \\ \text { TCGA-CN-5360-01 } & \mathrm{M} 0 \\ \text { TCGA-CN-5363-01 } & \mathrm{R} \\ \text { TCGA-CN-5364-01 } & \mathrm{M} 0 \\ \text { TCGA-CN-5365-01 } & \mathrm{M} 1 \\ \text { TCGA-CN-5366-01 } & \mathrm{R} \\ \text { TCGA-CN-5367-01 } & \mathrm{M} 0 \\ \text { TCGA-CN-5369-01 } & \mathrm{M} 0 \\ \text { TCGA-CN-5370-01 } & \mathrm{R} \\ \text { TCGA-CN-5373-01 } & \mathrm{M} 0 \\ \end{array}$




$\begin{array}{ll}\text { TCGA-CN-5374-01 } & \mathrm{R} \\ \text { TCGA-CN-6010-01 } & \mathrm{R} \\ \text { TCGA-CN-6011-01 } & \mathrm{M} 0 \\ \text { TCGA-CN-6012-01 } & \mathrm{M} 0 \\ \text { TCGA-CN-6013-01 } & \mathrm{R} \\ \text { TCGA-CN-6016-01 } & \mathrm{M} 0 \\ \text { TCGA-CN-6017-01 } & \mathrm{M} 0 \\ \text { TCGA-CN-6018-01 } & \mathrm{M} 0 \\ \text { TCGA-CN-6019-01 } & \mathrm{M} 0 \\ \text { TCGA-CN-6020-01 } & \mathrm{M} 0 \\ \text { TCGA-CN-6021-01 } & \mathrm{M} 0 \\ \text { TCGA-CN-6022-01 } & \mathrm{R} \\ \text { TCGA-CN-6023-01 } & \mathrm{M} 0 \\ \text { TCGA-CN-6024-01 } & \mathrm{R} \\ \text { TCGA-CN-6988-01 } & \mathrm{M} 0 \\ \text { TCGA-CN-6989-01 } & \mathrm{R} \\ \text { TCGA-CN-6992-01 } & \mathrm{M} 0 \\ \text { TCGA-CN-6994-01 } & \mathrm{M} 0 \\ \text { TCGA-CN-6995-01 } & \mathrm{M} 0 \\ \text { TCGA-CN-6996-01 } & \mathrm{R} \\ \text { TCGA-CN-6997-01 } & \mathrm{M} 0 \\ \text { TCGA-CN-6998-01 } & \mathrm{M} 0 \\ \text { TCGA-CN-A498-01 } & \mathrm{R} \\ \text { TCGA-CN-A49A-01 } & \mathrm{R} \\ \text { TCGA-CN-A642-01 } & \mathrm{M} 0 \\ \text { TCGA-CN-A6V3-01 } & \mathrm{M} 0 \\ \text { TCGA-CQ-5323-01 } & \mathrm{M} 0\end{array}$




$\begin{array}{ll}\text { TCGA-CQ-5324-01 } & \text { M0 } \\ \text { TCGA-CQ-5325-01 } & \text { R } \\ \text { TCGA-CQ-5326-01 } & \text { M0 } \\ \text { TCGA-CQ-5327-01 } & \text { M0 } \\ \text { TCGA-CQ-5329-01 } & \text { M0 } \\ \text { TCGA-CQ-5330-01 } & \text { M0 } \\ \text { TCGA-CQ-5331-01 } & \text { M0 } \\ \text { TCGA-CQ-5332-01 } & \text { M0 } \\ \text { TCGA-CQ-5333-01 } & \mathrm{R} \\ \text { TCGA-CQ-5334-01 } & \mathrm{R} \\ \text { TCGA-CQ-6218-01 } & \mathrm{M} 0 \\ \text { TCGA-CQ-6220-01 } & \mathrm{M} 0 \\ \text { TCGA-CQ-6221-01 } & \mathrm{M} 0 \\ \text { TCGA-CQ-6223-01 } & \mathrm{M} 0 \\ \text { TCGA-CQ-6224-01 } & \mathrm{M} 0 \\ \text { TCGA-CQ-6225-01 } & \mathrm{R} \\ \text { TCGA-CQ-6227-01 } & \mathrm{M} 0 \\ \text { TCGA-CQ-6228-01 } & \mathrm{R} \\ \text { TCGA-CQ-6229-01 } & \mathrm{M} 0 \\ \text { TCGA-CQ-7063-01 } & \mathrm{M} 0 \\ \text { TCGA-CQ-7065-01 } & \mathrm{R} \\ \text { TCGA-CQ-7067-01 } & \mathrm{M} 0 \\ \text { TCGA-CQ-7068-01 } & \mathrm{M} 0 \\ \text { TCGA-CQ-7069-01 } & \mathrm{M} 0 \\ \text { TCGA-CQ-7071-01 } & \mathrm{M} 0 \\ \text { TCGA-CQ-7072-01 } & \mathrm{M} 0 \\ \text { TCGA-CQ-A4C6-01 } & \mathrm{M} 0\end{array}$




$\begin{array}{ll}\text { TCGA-CQ-A4C7-01 } & \text { M0 } \\ \text { TCGA-CQ-A4C9-01 } & \text { R } \\ \text { TCGA-CQ-A4CB-01 } & \text { M0 } \\ \text { TCGA-CQ-A4CD-01 } & \text { M0 } \\ \text { TCGA-CQ-A4CE-01 } & \text { M0 } \\ \text { TCGA-CQ-A4CG-01 } & \text { M0 } \\ \text { TCGA-CQ-A4CH-01 } & \text { M0 } \\ \text { TCGA-CR-5243-01 } & \text { M0 } \\ \text { TCGA-CR-5247-01 } & \text { M0 } \\ \text { TCGA-CR-5248-01 } & \text { R } \\ \text { TCGA-CR-5249-01 } & \text { M0 } \\ \text { TCGA-CR-6467-01 } & \text { M0 } \\ \text { TCGA-CR-6470-01 } & \text { M0 } \\ \text { TCGA-CR-6471-01 } & \text { R } \\ \text { TCGA-CR-6472-01 } & \text { M0 } \\ \text { TCGA-CR-6473-01 } & \text { M0 } \\ \text { TCGA-CR-6474-01 } & \mathrm{R} \\ \text { TCGA-CR-6477-01 } & \text { M0 } \\ \text { TCGA-CR-6478-01 } & \text { M0 } \\ \text { TCGA-CR-6480-01 } & \text { M0 } \\ \text { TCGA-CR-6481-01 } & \text { M0 } \\ \text { TCGA-CR-6482-01 } & \text { M0 } \\ \text { TCGA-CR-6484-01 } & \text { M0 } \\ \text { TCGA-CR-6487-01 } & \text { M0 } \\ \text { TCGA-CR-6488-01 } & \text { M0 } \\ \text { TCGA-CR-6491-01 } & \text { M0 } \\ \text { TCGA-CR-6492-01 } & \text { M0 }\end{array}$




$\begin{array}{ll}\text { TCGA-CR-6493-01 } & \text { M0 } \\ \text { TCGA-CR-7364-01 } & \text { M0 } \\ \text { TCGA-CR-7365-01 } & \text { M0 } \\ \text { TCGA-CR-7367-01 } & \text { M0 } \\ \text { TCGA-CR-7368-01 } & \text { M0 } \\ \text { TCGA-CR-7369-01 } & \text { M0 } \\ \text { TCGA-CR-7370-01 } & \text { M0 } \\ \text { TCGA-CR-7371-01 } & \text { M0 } \\ \text { TCGA-CR-7372-01 } & \text { M0 } \\ \text { TCGA-CR-7373-01 } & \text { M0 } \\ \text { TCGA-CR-7374-01 } & \text { M0 } \\ \text { TCGA-CR-7376-01 } & \text { M0 } \\ \text { TCGA-CR-7377-01 } & \text { M0 } \\ \text { TCGA-CR-7379-01 } & \text { M0 } \\ \text { TCGA-CR-7380-01 } & \text { R } \\ \text { TCGA-CR-7382-01 } & \mathrm{R} \\ \text { TCGA-CR-7383-01 } & \mathrm{R} \\ \text { TCGA-CR-7385-01 } & \text { M0 } \\ \text { TCGA-CR-7386-01 } & \mathrm{R} \\ \text { TCGA-CR-7388-01 } & \mathrm{R} \\ \text { TCGA-CR-7389-01 } & \mathrm{M} 0 \\ \text { TCGA-CR-7390-01 } & \mathrm{M} 0 \\ \text { TCGA-CR-7391-01 } & \mathrm{M} 0 \\ \text { TCGA-CR-7392-01 } & \mathrm{M} 0 \\ \text { TCGA-CR-7393-01 } & \mathrm{M} 0 \\ \text { TCGA-CR-7394-01 } & \mathrm{M} 0 \\ \text { TCGA-CR-7395-01 } & \mathrm{M} 0\end{array}$




$\begin{array}{ll}\text { TCGA-CR-7397-01 } & \text { M0 } \\ \text { TCGA-CR-7398-01 } & \text { M0 } \\ \text { TCGA-CR-7399-01 } & \text { M0 } \\ \text { TCGA-CR-7401-01 } & \text { M0 } \\ \text { TCGA-CR-7402-01 } & \text { M0 } \\ \text { TCGA-CR-7404-01 } & \mathrm{R} \\ \text { TCGA-CV-5430-01 } & \mathrm{R} \\ \text { TCGA-CV-5432-01 } & \mathrm{M} 0 \\ \text { TCGA-CV-5434-01 } & \mathrm{R} \\ \text { TCGA-CV-5435-01 } & \mathrm{R} \\ \text { TCGA-CV-5436-01 } & \mathrm{M} 0 \\ \text { TCGA-CV-5439-01 } & \mathrm{R} \\ \text { TCGA-CV-5440-01 } & \mathrm{M} 0 \\ \text { TCGA-CV-5441-01 } & \mathrm{M} 0 \\ \text { TCGA-CV-5442-01 } & \mathrm{M} 0 \\ \text { TCGA-CV-5443-01 } & \mathrm{M} 0 \\ \text { TCGA-CV-5444-01 } & \mathrm{M} 0 \\ \text { TCGA-CV-5966-01 } & \mathrm{M} 0 \\ \text { TCGA-CV-5970-01 } & \mathrm{M} 0 \\ \text { TCGA-CV-5971-01 } & \mathrm{M} 0 \\ \text { TCGA-CV-5973-01 } & \mathrm{M} 0 \\ \text { TCGA-CV-5976-01 } & \mathrm{M} 0 \\ \text { TCGA-CV-5977-01 } & \mathrm{M} 0 \\ \text { TCGA-CV-5978-01 } & \mathrm{M} 0 \\ \text { TCGA-CV-5979-01 } & \mathrm{M} 0 \\ \text { TCGA-CV-6003-01 } & \mathrm{M} 0 \\ \text { TCGA-CV-6433-01 } & \mathrm{M} 0\end{array}$




$\begin{array}{ll}\text { TCGA-CV-6436-01 } & \text { M0 } \\ \text { TCGA-CV-6441-01 } & \text { M0 } \\ \text { TCGA-CV-6933-01 } & \text { M0 } \\ \text { TCGA-CV-6934-01 } & \text { M0 } \\ \text { TCGA-CV-6935-01 } & \text { M0 } \\ \text { TCGA-CV-6936-01 } & \text { M0 } \\ \text { TCGA-CV-6937-01 } & \text { M0 } \\ \text { TCGA-CV-6938-01 } & \text { M0 } \\ \text { TCGA-CV-6939-01 } & \text { M0 } \\ \text { TCGA-CV-6940-01 } & \text { M0 } \\ \text { TCGA-CV-6941-01 } & \text { M0 } \\ \text { TCGA-CV-6942-01 } & \text { M0 } \\ \text { TCGA-CV-6943-01 } & \text { M0 } \\ \text { TCGA-CV-6945-01 } & \text { M0 } \\ \text { TCGA-CV-6948-01 } & \text { M0 } \\ \text { TCGA-CV-6950-01 } & \text { M0 } \\ \text { TCGA-CV-6951-01 } & \text { M0 } \\ \text { TCGA-CV-6952-01 } & \text { M0 } \\ \text { TCGA-CV-6953-01 } & \text { M0 } \\ \text { TCGA-CV-6954-01 } & \text { M0 } \\ \text { TCGA-CV-6955-01 } & \text { M0 } \\ \text { TCGA-CV-6956-01 } & \text { M0 } \\ \text { TCGA-CV-6959-01 } & \text { M0 } \\ \text { TCGA-CV-6960-01 } & \text { M0 } \\ \text { TCGA-CV-6961-01 } & \text { M0 } \\ \text { TCGA-CV-6962-01 } & \text { M0 } \\ \text { TCGA-CV-7089-01 } & \text { M0 }\end{array}$




$\begin{array}{ll}\text { TCGA-CV-7090-01 } & \text { M0 } \\ \text { TCGA-CV-7091-01 } & \text { M0 } \\ \text { TCGA-CV-7095-01 } & \text { M0 } \\ \text { TCGA-CV-7097-01 } & \text { M0 } \\ \text { TCGA-CV-7099-01 } & \text { M0 } \\ \text { TCGA-CV-7100-01 } & \text { M0 } \\ \text { TCGA-CV-7101-01 } & \text { M0 } \\ \text { TCGA-CV-7102-01 } & \text { M0 } \\ \text { TCGA-CV-7103-01 } & \text { M0 } \\ \text { TCGA-CV-7104-01 } & \text { M0 } \\ \text { TCGA-CV-7177-01 } & \text { M0 } \\ \text { TCGA-CV-7178-01 } & \text { M0 } \\ \text { TCGA-CV-7180-01 } & \text { M0 } \\ \text { TCGA-CV-7183-01 } & \text { M0 } \\ \text { TCGA-CV-7235-01 } & \text { M0 } \\ \text { TCGA-CV-7236-01 } & \text { M0 } \\ \text { TCGA-CV-7238-01 } & \text { M0 } \\ \text { TCGA-CV-7242-01 } & \text { M0 } \\ \text { TCGA-CV-7243-01 } & \text { M0 } \\ \text { TCGA-CV-7245-01 } & \text { M0 } \\ \text { TCGA-CV-7247-01 } & \text { M0 } \\ \text { TCGA-CV-7248-01 } & \text { M0 } \\ \text { TCGA-CV-7250-01 } & \text { M0 } \\ \text { TCGA-CV-7252-01 } & \text { M0 } \\ \text { TCGA-CV-7253-01 } & \text { M0 } \\ \text { TCGA-CV-7254-01 } & \text { M0 } \\ \text { TCGA-CV-7255-01 } & \text { M0 }\end{array}$




$\begin{array}{ll}\text { TCGA-CV-7261-01 } & \text { M0 } \\ \text { TCGA-CV-7263-01 } & \text { M0 } \\ \text { TCGA-CV-7406-01 } & \text { M0 } \\ \text { TCGA-CV-7407-01 } & \text { M0 } \\ \text { TCGA-CV-7409-01 } & \text { M0 } \\ \text { TCGA-CV-7410-01 } & \text { M0 } \\ \text { TCGA-CV-7411-01 } & \text { M0 } \\ \text { TCGA-CV-7413-01 } & \text { M0 } \\ \text { TCGA-CV-7414-01 } & \text { M0 } \\ \text { TCGA-CV-7415-01 } & \text { M0 } \\ \text { TCGA-CV-7416-01 } & \text { M0 } \\ \text { TCGA-CV-7418-01 } & \text { M0 } \\ \text { TCGA-CV-7421-01 } & \text { M0 } \\ \text { TCGA-CV-7422-01 } & \text { M0 } \\ \text { TCGA-CV-7423-01 } & \text { M0 } \\ \text { TCGA-CV-7424-01 } & \text { M0 } \\ \text { TCGA-CV-7425-01 } & \text { M0 } \\ \text { TCGA-CV-7427-01 } & \text { M0 } \\ \text { TCGA-CV-7429-01 } & \text { M0 } \\ \text { TCGA-CV-7430-01 } & \text { M0 } \\ \text { TCGA-CV-7432-01 } & \text { M0 } \\ \text { TCGA-CV-7433-01 } & \text { M0 } \\ \text { TCGA-CV-7434-01 } & \text { M0 } \\ \text { TCGA-CV-7435-01 } & \text { M0 } \\ \text { TCGA-CV-7437-01 } & \text { M0 } \\ \text { TCGA-CV-7438-01 } & \text { M0 } \\ \text { TCGA-CV-7440-01 } & \text { M0 }\end{array}$




$\begin{array}{ll}\text { TCGA-CV-A45O-01 } & \text { M0 } \\ \text { TCGA-CV-A45P-01 } & \text { R } \\ \text { TCGA-CV-A45Q-01 } & \text { M0 } \\ \text { TCGA-CV-A45R-01 } & \text { M0 } \\ \text { TCGA-CV-A45T-01 } & \text { M0 } \\ \text { TCGA-CV-A45U-01 } & \text { M0 } \\ \text { TCGA-CV-A45V-01 } & \text { M0 } \\ \text { TCGA-CV-A45W-01 } & \text { M0 } \\ \text { TCGA-CV-A45X-01 } & \text { M0 } \\ \text { TCGA-CV-A45Y-01 } & \text { M0 } \\ \text { TCGA-CV-A45Z-01 } & \text { M0 } \\ \text { TCGA-CV-A460-01 } & \mathrm{R} \\ \text { TCGA-CV-A461-01 } & \text { M0 } \\ \text { TCGA-CV-A463-01 } & \text { M0 } \\ \text { TCGA-CV-A464-01 } & \text { M0 } \\ \text { TCGA-CV-A465-01 } & \text { M0 } \\ \text { TCGA-CV-A468-01 } & \text { M0 } \\ \text { TCGA-CV-A6JD-01 } & \text { M0 } \\ \text { TCGA-CV-A6JE-01 } & \text { M0 } \\ \text { TCGA-CV-A6JM-01 } & \mathrm{R} \\ \text { TCGA-CV-A6JN-01 } & \text { M0 } \\ \text { TCGA-CV-A6JO-01 } & \text { M0 } \\ \text { TCGA-CV-A6JT-01 } & \text { M0 } \\ \text { TCGA-CV-A6JU-01 } & \text { M0 } \\ \text { TCGA-CV-A6JY-01 } & \text { M0 } \\ \text { TCGA-CV-A6JZ-01 } & \text { R } \\ \text { TCGA-CV-A6K0-01 } & \text { M0 }\end{array}$




$\begin{array}{ll}\text { TCGA-CV-A6K1-01 } & \text { R } \\ \text { TCGA-CV-A6K2-01 } & \mathrm{R} \\ \text { TCGA-CX-7082-01 } & \mathrm{M} 0 \\ \text { TCGA-CX-7085-01 } & \mathrm{M} 0 \\ \text { TCGA-CX-7086-01 } & \mathrm{M} 0 \\ \text { TCGA-CX-7219-01 } & \mathrm{M} 0 \\ \text { TCGA-D6-6515-01 } & \mathrm{R} \\ \text { TCGA-D6-6516-01 } & \mathrm{M} 0 \\ \text { TCGA-D6-6517-01 } & \mathrm{M} 0 \\ \text { TCGA-D6-6823-01 } & \mathrm{M} 0 \\ \text { TCGA-D6-6824-01 } & \mathrm{M} 0 \\ \text { TCGA-D6-6825-01 } & \mathrm{M} 0 \\ \text { TCGA-D6-6826-01 } & \mathrm{M} 0 \\ \text { TCGA-D6-6827-01 } & \mathrm{M} 0 \\ \text { TCGA-D6-8568-01 } & \mathrm{M} 0 \\ \text { TCGA-D6-8569-01 } & \mathrm{M} 0 \\ \text { TCGA-D6-A4Z9-01 } & \mathrm{M} 0 \\ \text { TCGA-D6-A4ZB-01 } & \mathrm{M} 0 \\ \text { TCGA-D6-A6EK-01 } & \mathrm{M} 0 \\ \text { TCGA-D6-A6EM-01 } & \mathrm{M} 0 \\ \text { TCGA-D6-A6EO-01 } & \mathrm{M} 0 \\ \text { TCGA-D6-A6EP-01 } & \mathrm{M} 0 \\ \text { TCGA-D6-A6EQ-01 } & \mathrm{M} 0 \\ \text { TCGA-D6-A6ES-01 } & \mathrm{M} 0 \\ \text { TCGA-D6-A74Q-01 } & \mathrm{M} 0 \\ \text { TCGA-DQ-5624-01 } & \mathrm{M} 0 \\ \text { TCGA-DQ-5625-01 } & \mathrm{R}\end{array}$




$\begin{array}{ll}\text { TCGA-DQ-5629-01 } & \text { M0 } \\ \text { TCGA-DQ-5630-01 } & \text { M0 } \\ \text { TCGA-DQ-5631-01 } & \mathrm{R} \\ \text { TCGA-DQ-7588-01 } & \mathrm{R} \\ \text { TCGA-DQ-7589-01 } & \mathrm{M} 1 \\ \text { TCGA-DQ-7590-01 } & \mathrm{M} 0 \\ \text { TCGA-DQ-7591-01 } & \mathrm{M} 0 \\ \text { TCGA-DQ-7592-01 } & \mathrm{M} 0 \\ \text { TCGA-DQ-7593-01 } & \mathrm{M} 0 \\ \text { TCGA-DQ-7594-01 } & \mathrm{M} 0 \\ \text { TCGA-DQ-7595-01 } & \mathrm{M} 0 \\ \text { TCGA-DQ-7596-01 } & \mathrm{R} \\ \text { TCGA-F7-7848-01 } & \mathrm{M} 0 \\ \text { TCGA-F7-8489-01 } & \mathrm{M} 0 \\ \text { TCGA-F7-A50G-01 } & \mathrm{M} 0 \\ \text { TCGA-F7-A50I-01 } & \mathrm{M} 0 \\ \text { TCGA-F7-A50J-01 } & \mathrm{M} 0 \\ \text { TCGA-F7-A61S-01 } & \mathrm{R} \\ \text { TCGA-H7-7774-01 } & \mathrm{R} \\ \text { TCGA-H7-8501-01 } & \mathrm{M} 0 \\ \text { TCGA-H7-A6C4-01 } & \mathrm{M} 0 \\ \text { TCGA-HD-7229-01 } & \mathrm{M} 0 \\ \text { TCGA-HD-7753-01 } & \mathrm{M} 0 \\ \text { TCGA-HD-7754-01 } & \mathrm{M} 0 \\ \text { TCGA-HD-7831-01 } & \mathrm{M} 0 \\ \text { TCGA-HD-7832-01 } & \mathrm{M} 0 \\ \text { TCGA-HD-7917-01 } & \mathrm{M} 0\end{array}$




$\begin{array}{ll}\text { TCGA-HD-8224-01 } & \mathrm{R} \\ \text { TCGA-HD-8314-01 } & \mathrm{M} 0 \\ \text { TCGA-HD-A633-01 } & \mathrm{R} \\ \text { TCGA-HL-7533-01 } & \mathrm{M} 0 \\ \text { TCGA-IQ-7630-01 } & \mathrm{M} 0 \\ \text { TCGA-IQ-7631-01 } & \mathrm{M} 0 \\ \text { TCGA-IQ-7632-01 } & \mathrm{M} 0 \\ \text { TCGA-IQ-A61I-01 } & \mathrm{M} 0 \\ \text { TCGA-IQ-A61J-01 } & \mathrm{M} 0 \\ \text { TCGA-KU-A66S-01 } & \mathrm{R} \\ \text { TCGA-KU-A66T-01 } & \mathrm{R} \\ \text { TCGA-KU-A6H7-01 } & \mathrm{M} 0 \\ \text { TCGA-KU-A6H8-01 } & \mathrm{R} \\ \text { TCGA-MT-A51W-01 } & \mathrm{M} 0 \\ \text { TCGA-MT-A51X-01 } & \mathrm{M} 0 \\ \text { TCGA-MT-A67D-01 } & \mathrm{M} 0 \\ \text { TCGA-MT-A7BN-01 } & \mathrm{R} \\ \text { TCGA-MZ-A5BI-01 } & \mathrm{M} 0 \\ \text { TCGA-MZ-A6I9-01 } & \mathrm{R} \\ \text { TCGA-P3-A5Q6-01 } & \mathrm{R} \\ \text { TCGA-P3-A6T5-01 } & \mathrm{R} \\ \text { TCGA-P3-A6T6-01 } & \mathrm{R} \\ \text { TCGA-QK-A64Z-01 } & \mathrm{R} \\ \text { TCGA-QK-A6IF-01 } & \mathrm{R} \\ \text { TCGA-QK-A6IG-01 } & \mathrm{R} \\ \text { TCGA-QK-A6IH-01 } & \mathrm{R} \\ \text { TCGA-QK-A6II-01 } & \mathrm{R}\end{array}$




$\begin{array}{ll}\text { TCGA-QK-A6IJ-01 } & \text { M0 } \\ \text { TCGA-QK-A6V9-01 } & \text { M0 } \\ \text { TCGA-QK-A6VB-01 } & \text { M0 } \\ \text { TCGA-QK-A6VC-01 } & \text { M0 } \\ \text { TCGA-RS-A6TO-01 } & \text { R } \\ \text { TCGA-RS-A6TP-01 } & \text { M0 } \\ \text { TCGA-T2-A6WX-01 } & \text { M0 } \\ \text { TCGA-T2-A6WZ-01 } & \text { R } \\ \text { TCGA-T2-A6X0-01 } & \text { M0 } \\ \text { TCGA-T2-A6X2-01 } & \text { M0 } \\ \text { TCGA-TN-A7HI-01 } & \text { M0 } \\ \text { TCGA-TN-A7HJ-01 } & \text { M0 } \\ \text { TCGA-TN-A7HL-01 } & \text { M0 } \\ \text { TCGA-UF-A718-01 } & \text { M0 } \\ \text { TCGA-UF-A719-01 } & \text { M0 } \\ \text { TCGA-UF-A71A-01 } & \text { M0 } \\ \text { TCGA-UF-A71B-01 } & \text { M0 } \\ \text { TCGA-UF-A71D-01 } & \text { M0 } \\ \text { TCGA-UF-A71E-01 } & \text { M0 } \\ \text { TCGA-UF-A7J9-01 } & \text { M0 } \\ \text { TCGA-UF-A7JA-01 } & \text { M0 } \\ \text { TCGA-UF-A7JC-01 } & \text { M0 } \\ \text { TCGA-UF-A7JD-01 } & \text { M0 } \\ \text { TCGA-UF-A7JF-01 } & \text { M0 } \\ \text { TCGA-UF-A7JH-01 } & \text { M0 } \\ \text { TCGA-UF-A7JJ-01 } & \text { M0 } \\ \text { TCGA-UF-A7JK-01 } & \text { M0 }\end{array}$




$\begin{array}{ll}\text { TCGA-UF-A7JO-01 } & \text { M0 } \\ \text { TCGA-UF-A7JS-01 } & \mathrm{R} \\ \text { TCGA-UF-A7JT-01 } & \mathrm{M} 0 \\ \text { TCGA-UF-A7JV-01 } & \mathrm{M} 0 \\ \text { TCGA-WA-A7GZ-01 } & \mathrm{M} 0 \\ \text { TCGA-WA-A7H4-01 } & \mathrm{M} 0 \\ \text { TCGA-CV-5430-11 } & \mathrm{N} \\ \text { TCGA-CV-5431-11 } & \mathrm{N} \\ \text { TCGA-CV-5432-11 } & \mathrm{N} \\ \text { TCGA-CV-5434-11 } & \mathrm{N} \\ \text { TCGA-CV-5435-11 } & \mathrm{N} \\ \text { TCGA-CV-5436-11 } & \mathrm{N} \\ \text { TCGA-CV-5439-11 } & \mathrm{N} \\ \text { TCGA-CV-5440-11 } & \mathrm{N} \\ \text { TCGA-CV-5441-11 } & \mathrm{N} \\ \text { TCGA-CV-5442-11 } & \mathrm{N} \\ \text { TCGA-CV-5443-11 } & \mathrm{N} \\ \text { TCGA-CV-5444-11 } & \mathrm{N} \\ \text { TCGA-CV-5966-11 } & \mathrm{N} \\ \text { TCGA-CV-5970-11 } & \mathrm{N} \\ \text { TCGA-CV-5971-11 } & \mathrm{N} \\ \text { TCGA-CV-5973-11 } & \mathrm{N} \\ \text { TCGA-CV-5976-11 } & \mathrm{N} \\ \text { TCGA-CV-5977-11 } & \mathrm{N} \\ \text { TCGA-CV-5978-11 } & \mathrm{N} \\ \text { TCGA-CV-5979-11 } & \mathrm{N} \\ \text { TCGA-CV-6003-11 } & \mathrm{N}\end{array}$




$\begin{array}{ll}\text { TCGA-CV-6433-11 } & \text { N } \\ \text { TCGA-CV-6436-11 } & \text { N } \\ \text { TCGA-CV-6441-11 } & \text { N } \\ \text { TCGA-CV-6933-11 } & \text { N } \\ \text { TCGA-CV-6934-11 } & \text { N } \\ \text { TCGA-CV-6935-11 } & \text { N } \\ \text { TCGA-CV-6936-11 } & \text { N } \\ \text { TCGA-CV-6938-11 } & \text { N } \\ \text { TCGA-CV-6939-11 } & \text { N } \\ \text { TCGA-CV-6943-11 } & \text { N } \\ \text { TCGA-CV-6951-11 } & \text { N } \\ \text { TCGA-CV-6952-11 } & \text { N } \\ \text { TCGA-CV-6953-11 } & \text { N } \\ \text { TCGA-CV-6954-11 } & \text { N } \\ \text { TCGA-CV-6955-11 } & \text { N } \\ \text { TCGA-CV-6956-11 } & \text { N } \\ \text { TCGA-CV-6959-11 } & \text { N } \\ \text { TCGA-CV-6960-11 } & \text { N } \\ \text { TCGA-CV-6961-11 } & \text { N } \\ \text { TCGA-CV-6962-11 } & \text { N } \\ \text { TCGA-CV-7089-11 } & \mathrm{N} \\ \text { TCGA-CV-7091-11 } & \mathrm{N} \\ \text { TCGA-CV-7097-11 } & \mathrm{N} \\ \text { TCGA-CV-7101-11 } & \mathrm{N} \\ \text { TCGA-CV-7103-11 } & \mathrm{N} \\ \text { TCGA-CV-7177-11 } & \mathrm{N} \\ \text { TCGA-CV-7178-11 } & \mathrm{N}\end{array}$




$\begin{array}{ll}\text { TCGA-CV-7183-11 } & \text { N } \\ \text { TCGA-CV-7235-11 } & \mathrm{N} \\ \text { TCGA-CV-7238-11 } & \mathrm{N} \\ \text { TCGA-CV-7242-11 } & \mathrm{N} \\ \text { TCGA-CV-7245-11 } & \mathrm{N} \\ \text { TCGA-CV-7250-11 } & \mathrm{N} \\ \text { TCGA-CV-7252-11 } & \mathrm{N} \\ \text { TCGA-CV-7255-11 } & \mathrm{N} \\ \text { TCGA-CV-7261-11 } & \mathrm{N} \\ \text { TCGA-CV-7263-11 } & \mathrm{N} \\ \text { TCGA-CV-7406-11 } & \mathrm{N} \\ \text { TCGA-CV-7416-11 } & \mathrm{N} \\ \text { TCGA-CV-7423-11 } & \mathrm{N} \\ \text { TCGA-CV-7424-11 } & \mathrm{N} \\ \text { TCGA-CV-7425-11 } & \mathrm{N} \\ \text { TCGA-CV-7432-11 } & \mathrm{N} \\ \text { TCGA-CV-7434-11 } & \mathrm{N} \\ \text { TCGA-CV-7437-11 } & \mathrm{N} \\ \text { TCGA-CV-7438-11 } & \mathrm{N} \\ \text { TCGA-CV-7440-11 } & \mathrm{N} \\ \text { TCGA-H7-A6C5-11 } & \mathrm{N} \\ \text { TCGA-HD-8635-11 } & \mathrm{N} \\ \text { TCGA-HD-A6HZ-11 } & \mathrm{N} \\ \text { TCGA-HD-A6I0-11 } & \mathrm{N} \\ \text { TCGA-WA-A7GZ-11 } & \mathrm{N}\end{array}$


Supplementary Table S2: Oral tongue squamous cell carcinoma (OTSCC) and TCGA HNSC samples lacking cross-platform overlap, and their clinical attributes used for validation.

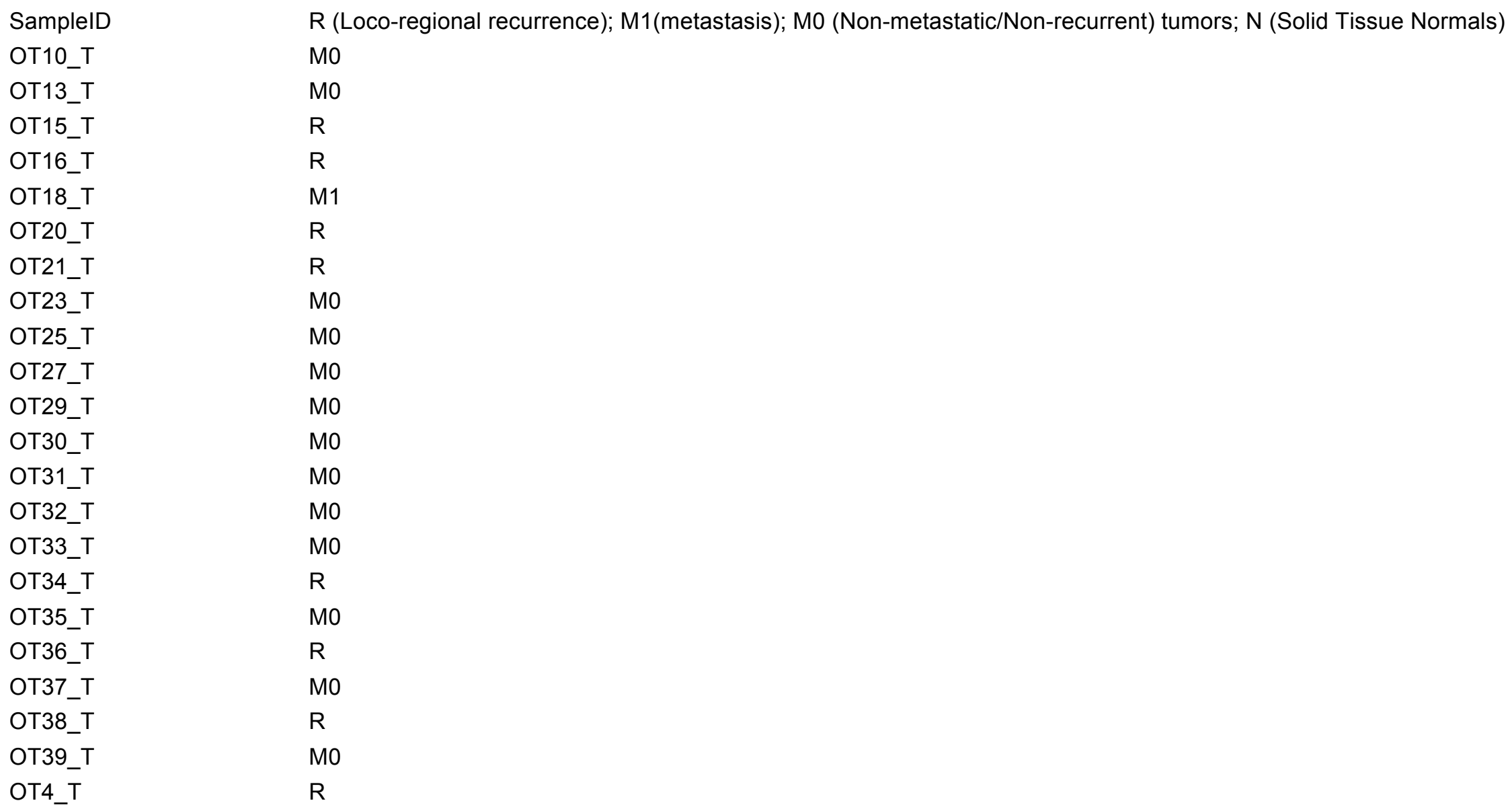




\begin{tabular}{|c|c|}
\hline OT40_T & $\mathrm{R}$ \\
\hline OT41_T & MO \\
\hline OT42_T & Mo \\
\hline OT43_T & MO \\
\hline OT45_T & Mo \\
\hline OT46_T & MO \\
\hline OT47_T & MO \\
\hline OT48_T & $\mathrm{R}$ \\
\hline OT49_T & $\mathrm{R}$ \\
\hline OT5_T & $\mathrm{R}$ \\
\hline OT50_T & M1 \\
\hline OT53_T & MO \\
\hline OT55_T & $\mathrm{R}$ \\
\hline OT7_T & Mo \\
\hline OT9_T & MO \\
\hline TCGA-BA-A6DE-01 & Mo \\
\hline TCGA-BA-A6DF-01 & Mo \\
\hline TCGA-BA-A6DF-01 & Mo \\
\hline TCGA-BA-A6DL-01 & Mo \\
\hline TCGA-BA-A8YP-01 & M0 \\
\hline TCGA-BA-A8YP-01 & Mo \\
\hline TCGA-BB-7864-01 & Mo \\
\hline TCGA-BB-7866-01 & Mo \\
\hline TCGA-BB-7866-01 & M0 \\
\hline TCGA-C9-A480-01 & M0 \\
\hline TCGA-CN-4722-01 & Mo \\
\hline TCGA-CN-4722-01 & Mo \\
\hline
\end{tabular}




$\begin{array}{ll}\text { TCGA-CN-4734-01 } & \text { M0 } \\ \text { TCGA-CN-4734-01 } & \text { M0 } \\ \text { TCGA-CN-5361-01 } & \text { M0 } \\ \text { TCGA-CN-5361-01 } & \text { M0 } \\ \text { TCGA-CQ-6219-01 } & \text { R } \\ \text { TCGA-CQ-6219-01 } & \text { R } \\ \text { TCGA-CQ-6222-01 } & \text { M0 } \\ \text { TCGA-CQ-6222-01 } & \text { M0 } \\ \text { TCGA-CQ-7063-01 } & \text { M0 } \\ \text { TCGA-CQ-7064-01 } & \text { M0 } \\ \text { TCGA-CQ-7064-01 } & \text { M0 } \\ \text { TCGA-CQ-A4C9-01 } & \text { M0 } \\ \text { TCGA-CQ-A4CA-01 } & \text { M0 } \\ \text { TCGA-CQ-A4CA-01 } & \text { M0 } \\ \text { TCGA-CR-5249-01 } & \text { M0 } \\ \text { TCGA-CR-5250-01 } & \text { M0 } \\ \text { TCGA-CR-5250-01 } & \text { M0 } \\ \text { TCGA-CV-5430-01 } & \text { M0 } \\ \text { TCGA-CV-5431-01 } & \text { M0 } \\ \text { TCGA-CV-5431-01 } & \text { M0 } \\ \text { TCGA-CV-7427-01 } & \text { M0 } \\ \text { TCGA-CV-7428-01 } & \text { M0 } \\ \text { TCGA-CV-7428-01 } & \text { M0 } \\ \text { TCGA-CV-7446-01 } & \text { M0 } \\ \text { TCGA-CV-7446-01 } & \text { M0 } \\ \text { TCGA-CV-7568-01 } & \text { M0 } \\ \text { TCGA-CV-7568-01 } & \text { M0 }\end{array}$




$\begin{array}{ll}\text { TCGA-F7-7848-01 } & \text { M0 } \\ \text { TCGA-F7-8298-01 } & \text { M0 } \\ \text { TCGA-F7-8298-01 } & \text { M0 } \\ \text { TCGA-H7-8501-01 } & \text { M0 } \\ \text { TCGA-H7-8502-01 } & \text { M0 } \\ \text { TCGA-H7-8502-01 } & \text { M0 } \\ \text { TCGA-H7-A6C4-01 } & \text { M0 } \\ \text { TCGA-H7-A6C5-01 } & \text { M0 } \\ \text { TCGA-H7-A6C5-01 } & \text { M0 } \\ \text { TCGA-IQ-A61J-01 } & \text { M0 } \\ \text { TCGA-IQ-A61K-01 } & \text { M0 } \\ \text { TCGA-IQ-A61K-01 } & \text { M0 } \\ \text { TCGA-IQ-A61L-01 } & \text { M0 } \\ \text { TCGA-IQ-A61L-01 } & \text { M0 } \\ \text { TCGA-KU-A6H7-06 } & \text { M0 } \\ \text { TCGA-KU-A6H7-06 } & \text { M0 } \\ \text { TCGA-QK-A6VC-01 } & \text { M0 } \\ \text { TCGA-QK-A8Z7-01 } & \text { M0 } \\ \text { TCGA-QK-A8Z7-01 } & \text { M1 } \\ \text { TCGA-QK-A8Z8-01 } & \text { M1 } \\ \text { TCGA-QK-A8Z8-01 } & \text { R } \\ \text { TCGA-QK-A8Z9-01 } & \text { R } \\ \text { TCGA-QK-A8Z9-01 } & \text { R } \\ \text { TCGA-QK-A8ZA-01 } & \text { M0 } \\ \text { TCGA-QK-A8ZB-0 } & \text { M0 } \\ \text { TCGA-QK-A8ZB-01 } & \text { M0 } \\ \text { TCGA-QK-AA3J-01 } & \text { M0 }\end{array}$




$\begin{array}{lc}\text { TCGA-QK-AA3J-01 } & \text { M0 } \\ \text { TCGA-UF-A71A-06 } & \text { M0 } \\ \text { TCGA-UF-A71A-06 } & \text { M0 } \\ \text { OT10_N } & \mathrm{N} \\ \text { OT13_N } & \mathrm{N} \\ \text { OT15_N } & \mathrm{N} \\ \text { OT16_N } & \mathrm{N} \\ \text { OT18_N } & \mathrm{N} \\ \text { OT20_N } & \mathrm{N} \\ \text { OT21_N } & \mathrm{N} \\ \text { OT23_N } & \mathrm{N} \\ \text { OT25_N } & \mathrm{N} \\ \text { OT27_N } & \mathrm{N} \\ \text { OT29_N } & \mathrm{N} \\ \text { OT30_N } & \mathrm{N} \\ \text { OT31_N } & \mathrm{N} \\ \text { OT32_N } & \mathrm{N} \\ \text { OT33_N } & \mathrm{N} \\ \text { OT34_N } & \mathrm{N} \\ \text { OT35_N } & \mathrm{N} \\ \text { OT36_N } & \mathrm{N} \\ \text { OT37_N } & \mathrm{N} \\ \text { OT38_N } & \mathrm{N} \\ \text { OT39_N } & \mathrm{N} \\ \text { OT4_N } & \mathrm{N} \\ \text { OT40_N } & \mathrm{N} \\ \text { OT41_N } & \mathrm{N}\end{array}$




$\begin{array}{lr}\text { OT42_N } & \text { N } \\ \text { OT43_N } & \text { N } \\ \text { OT45_N } & \text { N } \\ \text { OT46_N } & \text { N } \\ \text { OT47_N } & \text { N } \\ \text { OT48_N } & \text { N } \\ \text { OT49_N } & \text { N } \\ \text { OT5_N } & \text { N } \\ \text { OT50_N } & \text { N } \\ \text { OT53_N } & \text { N } \\ \text { OT55_N } & \text { N } \\ \text { OT7_N } & \text { N } \\ \text { OT9_N } & \\ & \end{array}$

\begin{tabular}{|l|l|}
\hline $\begin{array}{l}\text { Supplementary Table S3: Oral tongue squamous cell carcinoma (OTSCC) with all four events assayed within the same tumor, and their clinical attributes } \\
\text { used for validation. }\end{array}$ \\
\hline S & R (Loco-regional recurrence); M1(metastasis); M0 (Non-metastatic/Non-recurrent) tumors; N (Solid Tissue Normals). \\
a & \\
m & \\
I & \\
e & \\
I & M0 \\
\hline OT1_T & M0 \\
\hline OT11_T & M0 \\
\hline OT12_T & M0 \\
\hline OT14_T &
\end{tabular}




\begin{tabular}{|c|c|}
\hline OT17_T & MO \\
\hline OT19_T & MO \\
\hline OT2_T & M1 \\
\hline OT22_T & MO \\
\hline OT24_T & MO \\
\hline OT26_T & MO \\
\hline OT28_T & MO \\
\hline OT52_T & MO \\
\hline OT54_T & MO \\
\hline OT3_T & MO \\
\hline OT6_T & M0 \\
\hline OT8_T & M0 \\
\hline OT44_T & $\mathrm{R}$ \\
\hline OT51_T & M1 \\
\hline OT1_N & $\mathrm{N}$ \\
\hline OT11_N & $\mathrm{N}$ \\
\hline OT12_N & $\mathrm{N}$ \\
\hline OT14_N & $\mathrm{N}$ \\
\hline OT17_N & $\mathrm{N}$ \\
\hline OT19_N & $\mathrm{N}$ \\
\hline OT2_N & $\mathrm{N}$ \\
\hline OT22_N & $\mathrm{N}$ \\
\hline OT24_N & $\mathrm{N}$ \\
\hline OT26_N & $\mathrm{N}$ \\
\hline OT28_N & $\mathrm{N}$ \\
\hline OT52_N & $\mathrm{N}$ \\
\hline
\end{tabular}




\begin{tabular}{|l|l|}
\hline OT54_N & N \\
\hline OT3_N & N \\
\hline OT6_N & N \\
\hline OT8_N & N \\
\hline OT44_N & N \\
\hline OT51_N & N \\
\hline
\end{tabular}


Supplementary Table S4: Discovered events associated with Metastases and Recurrence in TCGA HNSCC, pathways mapped, confirmation and validation status.

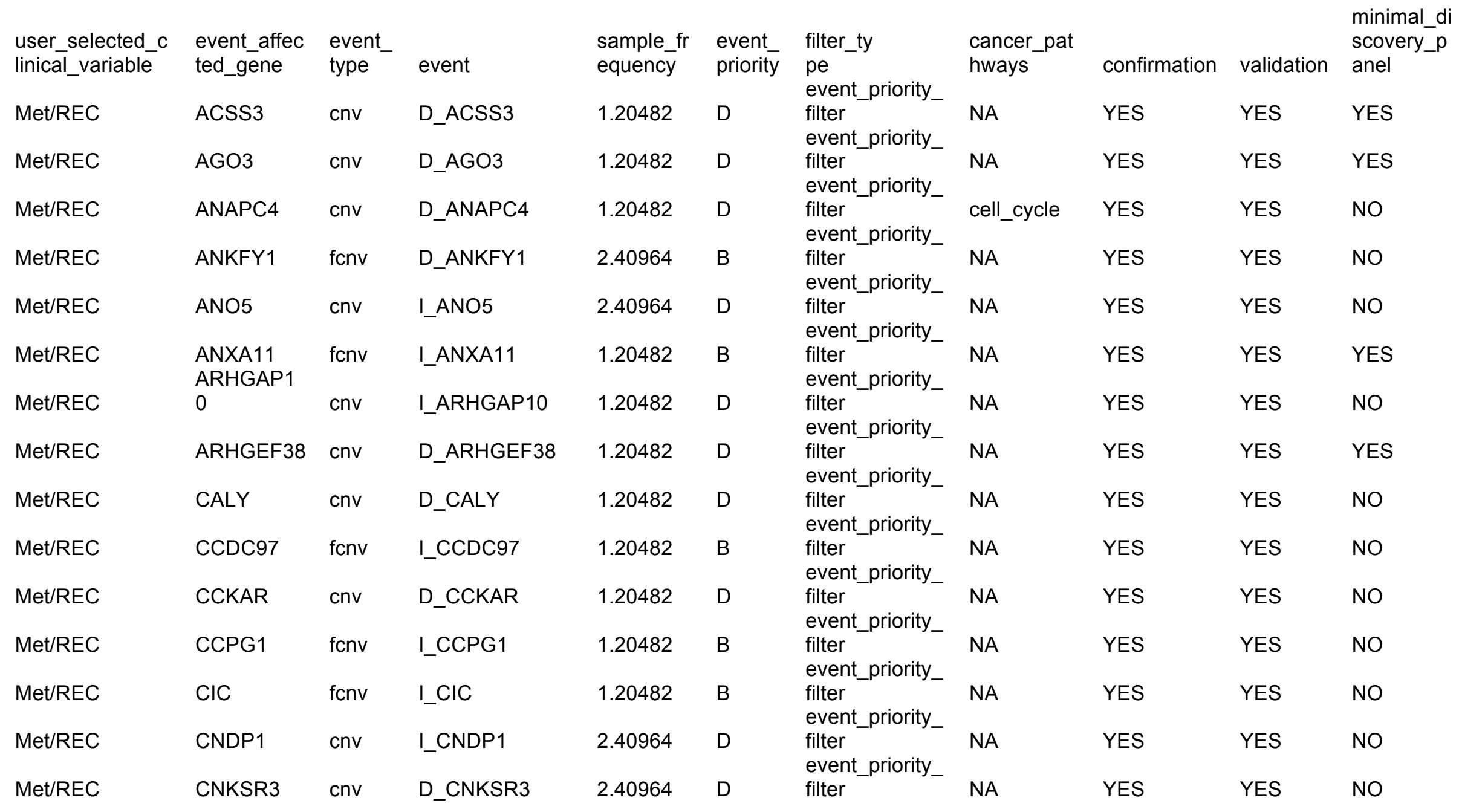




\begin{tabular}{|c|c|c|c|c|c|c|c|c|c|c|}
\hline Met/REC & COPS2 & fcnv & I_COPS2 & 1.20482 & B & $\begin{array}{l}\text { event_priority_ } \\
\text { filter } \\
\text { event priority }\end{array}$ & NA & YES & YES & NO \\
\hline Met/REC & CSRP3 & $\mathrm{cnv}$ & I_CSRP3 & 2.40964 & D & $\begin{array}{l}\text { filter } \\
\text { event_priority_ }\end{array}$ & NA & YES & YES & NO \\
\hline Met/REC & DBX1 & cnv & I_DBX1 & 2.40964 & D & $\begin{array}{l}\text { filter } \\
\text { event priority }\end{array}$ & NA & YES & YES & NO \\
\hline Met/REC & DCLK2 & cnv & I_DCLK2 & 1.20482 & D & $\begin{array}{l}\text { filter } \\
\text { event priority }\end{array}$ & NA & YES & YES & NO \\
\hline Met/REC & DOCK2 & cnv & D_DOCK2 & 1.20482 & D & $\begin{array}{l}\text { filter } \\
\text { event_priority }\end{array}$ & NA & YES & YES & NO \\
\hline Met/REC & DVL2 & fcnv & D_DVL2 & 1.20482 & B & $\begin{array}{l}\text { filter } \\
\text { event_priority_ }\end{array}$ & mtor_wnt & YES & YES & YES \\
\hline Met/REC & ECHS1 & $\mathrm{cnv}$ & D_ECHS1 & 1.20482 & D & $\begin{array}{l}\text { filter } \\
\text { event_priority_ }\end{array}$ & NA & YES & YES & NO \\
\hline Met/REC & ERF & fcnv & I_ERF & 1.20482 & B & $\begin{array}{l}\text { filter } \\
\text { event_priority }\end{array}$ & NA & YES & YES & NO \\
\hline Met/REC & FBXW7 & cnv & I_FBXW7 & 1.20482 & D & $\begin{array}{l}\text { filter } \\
\text { event_priority_ }\end{array}$ & $\begin{array}{l}\text { NA } \\
\text { mapk_pi3k }\end{array}$ & YES & YES & NO \\
\hline Met/REC & FGF10 & cnv & D_FGF10 & 1.20482 & D & $\begin{array}{l}\text { filter } \\
\text { event_priority_ }\end{array}$ & mapk_pi3k & YES & YES & YES \\
\hline Met/REC & FGFR4 & fcnv & I_FGFR4 & 1.20482 & B & $\begin{array}{l}\text { filter } \\
\text { event_priority_ }\end{array}$ & _akt & YES & YES & YES \\
\hline Met/REC & FRMPD4 & $\mathrm{cnv}$ & I_FRMPD4 & 2.40964 & D & $\begin{array}{l}\text { filter } \\
\text { event_priority_ }\end{array}$ & NA & YES & YES & YES \\
\hline Met/REC & FUOM & cnv & D_FUOM & 1.20482 & D & $\begin{array}{l}\text { filter } \\
\text { event_priority_ }\end{array}$ & NA & YES & YES & NO \\
\hline Met/REC & GAS2 & cnv & I_GAS2 & 2.40964 & D & $\begin{array}{l}\text { filter } \\
\text { event priority }\end{array}$ & NA & YES & YES & NO \\
\hline Met/REC & GBE1 & cnv & I_GBE1 & 1.20482 & D & $\begin{array}{l}\text { filter } \\
\text { event_priority__ }\end{array}$ & NA & YES & YES & NO \\
\hline Met/REC & GCSAML & $\mathrm{cnv}$ & D_GCSAML & 1.20482 & D & $\begin{array}{l}\text { filter } \\
\text { event_priority_ }\end{array}$ & NA & YES & YES & NO \\
\hline Met/REC & GNPDA2 & cnv & D_GNPDA2 & 3.61446 & D & $\begin{array}{l}\text { filter } \\
\text { event_priority_ }\end{array}$ & NA & YES & YES & NO \\
\hline Met/REC & GRXCR1 & cnv & D_GRXCR1 & 3.61446 & D & filter & NA & YES & YES & NO \\
\hline
\end{tabular}




\begin{tabular}{|c|c|c|c|c|c|c|c|c|c|c|}
\hline Met/REC & GTF2H1 & cnv & I_GTF2H1 & 2.40964 & D & $\begin{array}{l}\text { event_priority_ } \\
\text { filter } \\
\text { event priority }\end{array}$ & NA & YES & YES & NO \\
\hline Met/REC & GUF1 & cnv & D_GUF1 & 3.61446 & D & $\begin{array}{l}\text { filter } \\
\text { event_priority }\end{array}$ & NA & YES & YES & NO \\
\hline Met/REC & HK2 & cnv & D_HK2 & 1.20482 & D & $\begin{array}{l}\text { filter } \\
\text { event priority }\end{array}$ & NA & YES & YES & YES \\
\hline Met/REC & HTATIP2 & cnv & I_HTATIP2 & 2.40964 & D & $\begin{array}{l}\text { filter } \\
\text { event_priority__ }\end{array}$ & NA & YES & YES & NO \\
\hline Met/REC & IGSF22 & cnv & I_IGSF22 & 2.40964 & D & $\begin{array}{l}\text { filter } \\
\text { event_priority_ }\end{array}$ & NA & YES & YES & NO \\
\hline Met/REC & $\mathrm{KCNC} 1$ & $\mathrm{cnv}$ & I_KCNC1 & 2.40964 & D & $\begin{array}{l}\text { filter } \\
\text { event_priority_ }\end{array}$ & NA & YES & YES & NO \\
\hline Met/REC & LDHAL6A & $\mathrm{cnv}$ & I_LDHAL6A & 2.40964 & D & $\begin{array}{l}\text { filter } \\
\text { event_priority_ }\end{array}$ & NA & YES & YES & NO \\
\hline Met/REC & MAGI3 & cnv & I_MAGI3 & 1.20482 & D & $\begin{array}{l}\text { filter } \\
\text { event priority }\end{array}$ & NA & YES & YES & YES \\
\hline Met/REC & MAT1A & fenv & I_MAT1A & 1.20482 & B & $\begin{array}{l}\text { filter } \\
\text { event_priority_ }\end{array}$ & NA & YES & YES & NO \\
\hline Met/REC & MIR573 & cnv & I_MIR573 & 1.20482 & D & $\begin{array}{l}\text { filter } \\
\text { event_priority_ }\end{array}$ & NA & YES & YES & YES \\
\hline Met/REC & MMAA & cnv & I_MMAA & 1.20482 & D & $\begin{array}{l}\text { filter } \\
\text { event_priority_ }\end{array}$ & NA & YES & YES & NO \\
\hline Met/REC & MORC3 & fenv & I_MORC3 & 1.20482 & B & $\begin{array}{l}\text { filter } \\
\text { event_priority_ }\end{array}$ & NA & NO & NA & NO \\
\hline Met/REC & $\begin{array}{l}\text { MORF4L1 } \\
\text { MPHOSPH }\end{array}$ & fcnv & D_MORF4L1 & 1.20482 & B & $\begin{array}{l}\text { filter } \\
\text { event_priority_ }\end{array}$ & NA & YES & YES & YES \\
\hline Met/REC & 6 & cnv & D_MPHOSPH6 & 1.20482 & D & $\begin{array}{l}\text { filter } \\
\text { event priority }\end{array}$ & NA & YES & YES & YES \\
\hline Met/REC & MRGPRX2 & cnv & I_MRGPRX2 & 2.40964 & D & $\begin{array}{l}\text { filter } \\
\text { event_priority__ }\end{array}$ & NA & YES & YES & NO \\
\hline Met/REC & MTHFS & fcnv & D_MTHFS & 1.20482 & B & $\begin{array}{l}\text { filter } \\
\text { event_priority_ }\end{array}$ & NA & YES & YES & YES \\
\hline Met/REC & MUC15 & cnv & I_MUC15 & 2.40964 & D & $\begin{array}{l}\text { filter } \\
\text { event_priority_ }\end{array}$ & NA & YES & YES & NO \\
\hline Met/REC & MYO5C & fenv & I_MYO5C & 1.20482 & B & filter & NA & YES & YES & NO \\
\hline
\end{tabular}




\begin{tabular}{|c|c|c|c|c|c|c|c|c|c|c|}
\hline Met/REC & NAV2 & cnv & I_NAV2 & 2.40964 & D & $\begin{array}{l}\text { event_priority_ } \\
\text { filter } \\
\text { event priority }\end{array}$ & NA & YES & YES & NO \\
\hline Met/REC & NEDD4 & fcnv & I_NEDD4 & 1.20482 & B & $\begin{array}{l}\text { filter } \\
\text { event_priority_ }\end{array}$ & NA & YES & YES & NO \\
\hline Met/REC & NELL1 & cnv & I_NELL1 & 2.40964 & D & $\begin{array}{l}\text { filter } \\
\text { event priority }\end{array}$ & NA & YES & YES & NO \\
\hline Met/REC & NLRP3 & cnv & D_NLRP3 & 1.20482 & D & $\begin{array}{l}\text { filter } \\
\text { event_priority__ }\end{array}$ & NA & YES & YES & NO \\
\hline Met/REC & NNT & cnv & D_NNT & 1.20482 & D & $\begin{array}{l}\text { filter } \\
\text { event_priority }\end{array}$ & NA & YES & YES & YES \\
\hline Met/REC & NR3C2 & $\mathrm{cnv}$ & I_NR3C2 & 1.20482 & D & $\begin{array}{l}\text { filter } \\
\text { event_priority_ }\end{array}$ & NA & YES & YES & NO \\
\hline Met/REC & NSUN5 & fcnv & D_NSUN5 & 1.20482 & B & $\begin{array}{l}\text { filter } \\
\text { event_priority_ }\end{array}$ & NA & YES & YES & NO \\
\hline Met/REC & OR2B11 & cnv & D_OR2B11 & 1.20482 & D & $\begin{array}{l}\text { filter } \\
\text { event priority }\end{array}$ & NA & YES & YES & NO \\
\hline Met/REC & OR2C3 & cnv & D_OR2C3 & 1.20482 & D & $\begin{array}{l}\text { filter } \\
\text { event priority }\end{array}$ & NA & YES & YES & NO \\
\hline Met/REC & OXCT1 & cnv & D_OXCT1 & 1.20482 & D & $\begin{array}{l}\text { filter } \\
\text { event_priority_ }\end{array}$ & NA & YES & YES & YES \\
\hline Met/REC & PFKM & fcnv & D_PFKM & 1.20482 & B & $\begin{array}{l}\text { filter } \\
\text { event_priority_ }\end{array}$ & NA & YES & YES & NO \\
\hline Met/REC & PLAUR & fcnv & I_PLAUR & 1.20482 & B & $\begin{array}{l}\text { filter } \\
\text { event_priority_ }\end{array}$ & NA & YES & YES & YES \\
\hline Met/REC & PRAP1 & cnv & D_PRAP1 & 1.20482 & D & $\begin{array}{l}\text { filter } \\
\text { event_priority_ }\end{array}$ & NA & YES & YES & NO \\
\hline Met/REC & PRMT3 & cnv & I_PRMT3 & 2.40964 & D & $\begin{array}{l}\text { filter } \\
\text { event priority }\end{array}$ & NA & YES & YES & NO \\
\hline Met/REC & PTPN5 & cnv & I_PTPN5 & 2.40964 & D & $\begin{array}{l}\text { filter } \\
\text { event_priority__ }\end{array}$ & mapk & YES & YES & NO \\
\hline Met/REC & PTPRR & cnv & D_PTPRR & 1.20482 & D & $\begin{array}{l}\text { filter } \\
\text { event_priority_ }\end{array}$ & mapk & NO & NA & NO \\
\hline Met/REC & RAB27A & fcnv & I_RAB27A & 1.20482 & B & $\begin{array}{l}\text { filter } \\
\text { event_priority_ }\end{array}$ & NA & YES & YES & NO \\
\hline Met/REC & RERGL & cnv & D_RERGL & 1.20482 & D & filter & NA & YES & YES & YES \\
\hline
\end{tabular}




\begin{tabular}{|c|c|c|c|c|c|c|c|c|c|c|}
\hline Met/REC & RFC2 & fenv & D_RFC2 & 1.20482 & B & $\begin{array}{l}\text { event_priority_ } \\
\text { filter } \\
\text { event_priority_ }\end{array}$ & NA & YES & YES & NO \\
\hline Met/REC & RICTOR & cnv & D_RICTOR & 1.20482 & D & $\begin{array}{l}\text { filter } \\
\text { event_priority }\end{array}$ & mtor & YES & YES & YES \\
\hline Met/REC & RPAP2 & cnv & D_RPAP2 & 1.20482 & D & $\begin{array}{l}\text { filter } \\
\text { event priority }\end{array}$ & NA & YES & YES & YES \\
\hline Met/REC & RPL26L1 & fcnv & I_RPL26L1 & 1.20482 & B & $\begin{array}{l}\text { filter } \\
\text { event_priority_}\end{array}$ & NA & YES & YES & YES \\
\hline Met/REC & RPS3A & $\mathrm{cnv}$ & I_RPS3A & 1.20482 & D & $\begin{array}{l}\text { filter } \\
\text { event_priority_ }\end{array}$ & NA & YES & YES & NO \\
\hline Met/REC & SAA4 & $\mathrm{cnv}$ & I_SAA4 & 2.40964 & D & $\begin{array}{l}\text { filter } \\
\text { event_priority_ }\end{array}$ & NA & YES & YES & NO \\
\hline Met/REC & SAAL1 & cnv & I_SAAL1 & 2.40964 & D & $\begin{array}{l}\text { filter } \\
\text { event_priority_ }\end{array}$ & NA & YES & YES & NO \\
\hline Met/REC & SEL1L3 & $\mathrm{cnv}$ & D_SEL1L3 & 1.20482 & D & $\begin{array}{l}\text { filter } \\
\text { event_priority_ }\end{array}$ & NA & YES & YES & NO \\
\hline Met/REC & SENP1 & fenv & D_SENP1 & 1.20482 & B & $\begin{array}{l}\text { filter } \\
\text { event_priority_ }\end{array}$ & NA & YES & YES & NO \\
\hline Met/REC & SERGEF & $\mathrm{cnv}$ & I_SERGEF & 2.40964 & $\mathrm{D}$ & $\begin{array}{l}\text { filter } \\
\text { event_priority_}\end{array}$ & NA & YES & YES & NO \\
\hline Met/REC & SH3D19 & $\mathrm{cnv}$ & I_SH3D19 & 1.20482 & D & $\begin{array}{l}\text { filter } \\
\text { event_priority_ }\end{array}$ & NA & YES & YES & NO \\
\hline Met/REC & SLC17A6 & cnv & I_SLC17A6 & 2.40964 & D & $\begin{array}{l}\text { filter } \\
\text { event_priority_}\end{array}$ & NA & YES & YES & NO \\
\hline Met/REC & SLC34A2 & cnv & D_SLC34A2 & 1.20482 & D & $\begin{array}{l}\text { filter } \\
\text { event priority }\end{array}$ & NA & YES & YES & NO \\
\hline Met/REC & SLC5A12 & $\mathrm{cnv}$ & I_SLC5A12 & 2.40964 & D & $\begin{array}{l}\text { filter } \\
\text { event_priority__ }\end{array}$ & NA & YES & YES & NO \\
\hline Met/REC & SMAD1 & $\mathrm{cnv}$ & I_SMAD1 & 1.20482 & D & $\begin{array}{l}\text { filter } \\
\text { event_priority_ }\end{array}$ & $\operatorname{tg} f$ & YES & YES & NO \\
\hline Met/REC & SMIM20 & cnv & D_SMIM20 & 1.20482 & $\mathrm{D}$ & $\begin{array}{l}\text { filter } \\
\text { event_priority_ }\end{array}$ & NA & YES & YES & NO \\
\hline Met/REC & SMIM21 & cnv & I_SMIM21 & 2.40964 & D & $\begin{array}{l}\text { filter } \\
\text { event_priority_}\end{array}$ & NA & YES & YES & NO \\
\hline Met/REC & SPTY2D1 & cnv & I_SPTY2D1 & 2.40964 & D & filter & NA & YES & YES & NO \\
\hline
\end{tabular}




\begin{tabular}{|c|c|c|c|c|c|c|c|c|c|c|}
\hline Met/REC & SRBD1 & cnv & D_SRBD1 & 1.20482 & D & $\begin{array}{l}\text { event_priority_ } \\
\text { filter } \\
\text { event priority }\end{array}$ & NA & YES & YES & NO \\
\hline Met/REC & SSBP2 & cnv & D_SSBP2 & 2.40964 & D & $\begin{array}{l}\text { filter } \\
\text { event_priority_ }\end{array}$ & NA & YES & YES & NO \\
\hline Met/REC & SVIP & cnv & I_SVIP & 2.40964 & D & $\begin{array}{l}\text { filter } \\
\text { event priority }\end{array}$ & NA & YES & YES & NO \\
\hline Met/REC & SYDE2 & cnv & D_SYDE2 & 2.40964 & D & $\begin{array}{l}\text { filter } \\
\text { event_priority__ }\end{array}$ & NA & YES & YES & NO \\
\hline Met/REC & TBC1D19 & cnv & D_TBC1D19 & 1.20482 & D & $\begin{array}{l}\text { filter } \\
\text { event_priority__ }\end{array}$ & NA & YES & YES & NO \\
\hline Met/REC & TMEM154 & $\mathrm{cnv}$ & I_TMEM154 & 1.20482 & D & $\begin{array}{l}\text { filter } \\
\text { event_priority_ }\end{array}$ & NA & YES & YES & NO \\
\hline Met/REC & TMEM155 & fcnv & I_TMEM155 & 1.20482 & B & $\begin{array}{l}\text { filter } \\
\text { event_priority_ }\end{array}$ & NA & YES & YES & NO \\
\hline Met/REC & TMEM86A & cnv & I_TMEM86A & 2.40964 & D & $\begin{array}{l}\text { filter } \\
\text { event priority }\end{array}$ & NA & YES & YES & NO \\
\hline Met/REC & TPH1 & cnv & I_TPH1 & 2.40964 & D & $\begin{array}{l}\text { filter } \\
\text { event_priority_ }\end{array}$ & NA & YES & YES & NO \\
\hline Met/REC & TSG101 & $\mathrm{cnv}$ & I_TSG101 & 2.40964 & D & $\begin{array}{l}\text { filter } \\
\text { event_priority_ }\end{array}$ & NA & YES & YES & NO \\
\hline Met/REC & TSHZ1 & cnv & I_TSHZ1 & 2.40964 & D & $\begin{array}{l}\text { filter } \\
\text { event_priority_ }\end{array}$ & NA & YES & YES & NO \\
\hline Met/REC & UEVLD & $\mathrm{cnv}$ & I_UEVLD & 2.40964 & D & $\begin{array}{l}\text { filter } \\
\text { event_priority_ }\end{array}$ & NA & YES & YES & NO \\
\hline Met/REC & USP32 & cnv & D_USP32 & 1.20482 & D & $\begin{array}{l}\text { filter } \\
\text { event priority }\end{array}$ & NA & YES & YES & YES \\
\hline Met/REC & USP8 & fenv & I_USP8 & 1.20482 & B & $\begin{array}{l}\text { filter } \\
\text { event priority }\end{array}$ & NA & YES & YES & NO \\
\hline Met/REC & YIPF7 & cnv & D_YIPF7 & 3.61446 & D & $\begin{array}{l}\text { filter } \\
\text { event_priority_ }\end{array}$ & NA & YES & YES & NO \\
\hline Met/REC & ZADH2 & $\mathrm{cnv}$ & I_ZADH2 & 2.40964 & D & $\begin{array}{l}\text { filter } \\
\text { event_priority_}\end{array}$ & NA & YES & YES & NO \\
\hline Met/REC & ZBTB7A & fcnv & I_ZBTB7A & 1.20482 & B & $\begin{array}{l}\text { filter } \\
\text { event_priority_ }\end{array}$ & NA & YES & YES & YES \\
\hline Met/REC & $\mathrm{ZCCHC} 4$ & cnv & D_ZCCHC4 & 1.20482 & D & filter & NA & YES & YES & NO \\
\hline
\end{tabular}




\begin{tabular}{|c|c|c|c|c|c|c|c|c|c|c|}
\hline Met/REC & ZDHHC13 & cnv & I_ZDHHC13 & 2.40964 & D & $\begin{array}{l}\text { event_priority_ } \\
\text { filter } \\
\text { event priority }\end{array}$ & NA & YES & YES & NO \\
\hline Met/REC & ZNF407 & cnv & I_ZNF407 & 2.40964 & D & $\begin{array}{l}\text { filter } \\
\text { event_priority_ }\end{array}$ & NA & YES & YES & NO \\
\hline Met/REC & ZNF511 & cnv & D_ZNF511 & 1.20482 & D & $\begin{array}{l}\text { filter } \\
\text { event_priority_ }\end{array}$ & NA & YES & YES & NO \\
\hline Met/REC & ZNF827 & cnv & I_ZNF827 & 1.20482 & D & $\begin{array}{l}\text { filter } \\
\text { event_priority__ }\end{array}$ & NA & YES & YES & NO \\
\hline Met/REC & ACAD8 & mut & ACAD8 & 3.61446 & A & $\begin{array}{l}\text { filter } \\
\text { sample_freque }\end{array}$ & NA & NO & NA & NO \\
\hline Met/REC & AIMP1 & expr & AIMP1 & 7.22892 & NA & $\begin{array}{l}\text { ncy_filter } \\
\text { event_priority_ }\end{array}$ & NA & YES & YES & YES \\
\hline Met/REC & C1QA & expr & C1QA & 4.81928 & $\mathrm{E}$ & $\begin{array}{l}\text { filter } \\
\text { event_priority_ }\end{array}$ & NA & NO & NA & NO \\
\hline Met/REC & C1QB & expr & C1QB & 4.81928 & $\mathrm{E}$ & $\begin{array}{l}\text { filter } \\
\text { event priority }\end{array}$ & NA & NO & NA & NO \\
\hline Met/REC & CHPF2 & expr & CHPF2 & 6.0241 & $\mathrm{E}$ & $\begin{array}{l}\text { filter } \\
\text { sample_freque }\end{array}$ & NA & NO & NA & NO \\
\hline Met/REC & CHPF2 & expr & CHPF2 & 6.0241 & NA & $\begin{array}{l}\text { ncy_filter } \\
\text { event_priority_ }\end{array}$ & NA & NO & NA & NO \\
\hline Met/REC & $\mathrm{CIC}$ & expr & $\mathrm{CIC}$ & 7.22892 & E & $\begin{array}{l}\text { filter } \\
\text { sample_freque }\end{array}$ & NA & YES & YES & YES \\
\hline Met/REC & $\mathrm{CIC}$ & expr & $\mathrm{CIC}$ & 7.22892 & NA & $\begin{array}{l}\text { ncy_filter } \\
\text { event_priority_ }\end{array}$ & NA & YES & YES & YES \\
\hline Met/REC & CTU1 & expr & CTU1 & 4.81928 & $E$ & $\begin{array}{l}\text { filter } \\
\text { sample freque }\end{array}$ & NA & YES & YES & YES \\
\hline Met/REC & CTU1 & expr & CTU1 & 4.81928 & NA & $\begin{array}{l}\text { ncy_filter } \\
\text { sample freque }\end{array}$ & NA & YES & YES & YES \\
\hline Met/REC & $\mathrm{DOHH}$ & expr & $\mathrm{DOHH}$ & 7.22892 & NA & $\begin{array}{l}\text { ncy_filtēer } \\
\text { event_priority__ }\end{array}$ & NA & YES & YES & YES \\
\hline Met/REC & DQX1 & expr & DQX1 & 4.81928 & $E$ & $\begin{array}{l}\text { filter } \\
\text { sample_freque }\end{array}$ & NA & NO & NA & NO \\
\hline Met/REC & DQX1 & expr & DQX1 & 4.81928 & NA & $\begin{array}{l}\text { ncy_filter } \\
\text { event_priority_ }\end{array}$ & NA & NO & NA & NO \\
\hline Met/REC & ECE2 & expr & ECE2 & 6.0241 & E & filter & NA & NO & NA & NO \\
\hline
\end{tabular}




\begin{tabular}{|c|c|c|c|c|c|c|c|c|c|c|}
\hline Met/REC & ECE2 & expr & ECE2 & 6.0241 & NA & $\begin{array}{l}\text { sample_freque } \\
\text { ncy_filter } \\
\text { event_priority }\end{array}$ & NA & NO & NA & NO \\
\hline Met/REC & EEF1B2 & mut & EEF1B2 & 1.20482 & A & $\begin{array}{l}\text { filter } \\
\text { event_priority_ }\end{array}$ & NA & YES & YES & NO \\
\hline Met/REC & FAM102A & expr & FAM102A & 4.81928 & $E$ & $\begin{array}{l}\text { filter } \\
\text { sample_freque }\end{array}$ & NA & NO & NA & NO \\
\hline Met/REC & FAM102A & expr & FAM102A & 4.81928 & NA & $\begin{array}{l}\text { ncy_filter } \\
\text { event_priority__ }\end{array}$ & NA & NO & NA & NO \\
\hline Met/REC & FAM134C & expr & FAM134C & 6.0241 & $\mathrm{E}$ & $\begin{array}{l}\text { filter } \\
\text { sample_freque }\end{array}$ & NA & YES & YES & YES \\
\hline Met/REC & FAM134C & expr & FAM134C & 6.0241 & NA & $\begin{array}{l}\text { ncy_filter } \\
\text { event_priority_ }\end{array}$ & NA & YES & YES & YES \\
\hline Met/REC & FOXO4 & expr & FOXO4 & 6.0241 & $\mathrm{E}$ & $\begin{array}{l}\text { filter } \\
\text { event_priority_ }\end{array}$ & NA & NO & NA & NO \\
\hline Met/REC & GMFB & expr & GMFB & 6.0241 & $\mathrm{E}$ & $\begin{array}{l}\text { filter } \\
\text { event priority }\end{array}$ & NA & YES & YES & YES \\
\hline Met/REC & GTF2IRD1 & expr & GTF2IRD1 & 4.81928 & $\mathrm{E}$ & $\begin{array}{l}\text { filter } \\
\text { event_priority }\end{array}$ & $\begin{array}{l}\text { NA } \\
\text { ecm_focal_} \\
\text { adhesion_p }\end{array}$ & NO & NA & NO \\
\hline Met/REC & ITGA9 & mut & ITGA9 & 1.20482 & A & $\begin{array}{l}\text { filter } \\
\text { event_priority_ }\end{array}$ & i3k_akt & YES & YES & YES \\
\hline Met/REC & KLF4 & mut & KLF4 & 1.20482 & A & $\begin{array}{l}\text { filter } \\
\text { event_priority__ }\end{array}$ & NA & YES & YES & NO \\
\hline Met/REC & KLHDC7B & expr & KLHDC7B & 6.0241 & $\mathrm{E}$ & $\begin{array}{l}\text { filter } \\
\text { sample_freque }\end{array}$ & NA & NO & NA & NO \\
\hline Met/REC & KLHDC7B & expr & KLHDC7B & 6.0241 & NA & $\begin{array}{l}\text { ncy_filter } \\
\text { event_priority_ }\end{array}$ & NA & NO & NA & NO \\
\hline Met/REC & LIMS1 & expr & LIMS1 & 6.0241 & $E$ & $\begin{array}{l}\text { filter } \\
\text { sample_freque }\end{array}$ & NA & YES & YES & YES \\
\hline Met/REC & LIMS1 & expr & LIMS1 & 6.0241 & NA & $\begin{array}{l}\text { ncy_filter } \\
\text { event_priority_}\end{array}$ & NA & YES & YES & YES \\
\hline Met/REC & MORC2 & expr & MORC2 & 4.81928 & $\mathrm{E}$ & $\begin{array}{l}\text { filter } \\
\text { sample_freque }\end{array}$ & NA & NO & NA & NO \\
\hline Met/REC & MORC2 & expr & MORC2 & 4.81928 & NA & ncy_filter & NA & NO & NA & NO \\
\hline
\end{tabular}




\begin{tabular}{|c|c|c|c|c|c|c|c|c|c|c|}
\hline Met/REC & MRS2 & expr & MRS2 & 7.22892 & NA & $\begin{array}{l}\text { sample_freque } \\
\text { ncy_filter } \\
\text { event priority }\end{array}$ & NA & NO & NA & NO \\
\hline Met/REC & NPM3 & expr & NPM3 & 7.22892 & E & $\begin{array}{l}\text { filter } \\
\text { sample_freque }\end{array}$ & NA & NO & NA & NO \\
\hline Met/REC & NPM3 & expr & NPM3 & 7.22892 & NA & ncy_filter & $\begin{array}{l}\text { NA } \\
\text { apoptosis__ } \\
\text { mapk_mtor }\end{array}$ & NO & NA & NO \\
\hline Met/REC & NRAS & mut & NRAS & 1.20482 & A & $\begin{array}{l}\text { event_priority_ } \\
\text { filter } \\
\text { event priority }\end{array}$ & $\underset{\text { vegf }}{\text { pi3k_akt_ }}$ & YES & YES & YES \\
\hline Met/REC & PCBP1 & mut & PCBP1 & 2.40964 & A & $\begin{array}{l}\text { filter } \\
\text { event_priority }\end{array}$ & NA & YES & YES & NO \\
\hline Met/REC & PGS1 & expr & PGS1 & 4.81928 & $\mathrm{E}$ & $\begin{array}{l}\text { filter } \\
\text { sample_freque }\end{array}$ & NA & NO & NA & NO \\
\hline Met/REC & PGS1 & expr & PGS1 & 4.81928 & NA & $\begin{array}{l}\text { ncy_filter } \\
\text { event_priority_ }\end{array}$ & NA & NO & NA & NO \\
\hline Met/REC & PJA2 & expr & PJA2 & 8.43373 & $\mathrm{E}$ & $\begin{array}{l}\text { filter } \\
\text { sample freque }\end{array}$ & NA & NO & NA & NO \\
\hline Met/REC & PJA2 & expr & PJA2 & 8.43373 & NA & $\begin{array}{l}\text { ncy_filter } \\
\text { event_priority_ }\end{array}$ & NA & NO & NA & NO \\
\hline Met/REC & PKP4 & expr & PKP4 & 7.22892 & E & $\begin{array}{l}\text { filter } \\
\text { sample_freque }\end{array}$ & NA & NO & NA & NO \\
\hline Met/REC & PKP4 & expr & PKP4 & 7.22892 & NA & $\begin{array}{l}\text { ncy_filter } \\
\text { sample_freque }\end{array}$ & NA & NO & NA & NO \\
\hline Met/REC & PPIAL4C & expr & PPIAL4C & 7.22892 & NA & $\begin{array}{l}\text { ncy_filter } \\
\text { event_priority_ }\end{array}$ & NA & YES & YES & YES \\
\hline Met/REC & PPIC & expr & PPIC & 4.81928 & $\mathrm{E}$ & $\begin{array}{l}\text { filter } \\
\text { sample_freque }\end{array}$ & NA & NO & NA & NO \\
\hline Met/REC & REXO2 & expr & REXO2 & 6.0241 & NA & $\begin{array}{l}\text { ncy_filtēer } \\
\text { event_priority__ }\end{array}$ & NA & NO & NA & NO \\
\hline Met/REC & RNF139 & expr & RNF139 & 7.22892 & $E$ & $\begin{array}{l}\text { filter } \\
\text { sample_freque }\end{array}$ & NA & NO & NA & NO \\
\hline Met/REC & RNF139 & expr & RNF139 & 7.22892 & NA & $\begin{array}{l}\text { ncy_filter } \\
\text { sample_freque }\end{array}$ & NA & NO & NA & NO \\
\hline Met/REC & SFRP1 & expr & SFRP1 & 4.81928 & NA & ncy_filter & wnt & NO & NA & NO \\
\hline
\end{tabular}




\begin{tabular}{|c|c|c|c|c|c|c|c|c|c|c|}
\hline Met/REC & SIGLEC1 & expr & SIGLEC1 & 3.61446 & E & $\begin{array}{l}\text { event_priority_ } \\
\text { filter } \\
\text { event_priority_ }\end{array}$ & NA & NO & NA & NO \\
\hline Met/REC & SLC9A5 & expr & SLC9A5 & 4.81928 & E & $\begin{array}{l}\text { filter } \\
\text { sample freque }\end{array}$ & NA & NO & NA & NO \\
\hline Met/REC & SLC9A5 & expr & SLC9A5 & 4.81928 & NA & $\begin{array}{l}\text { ncy_filter } \\
\text { event_priority__ }\end{array}$ & $\begin{array}{l}\text { NA } \\
\text { cell_cycle_t }\end{array}$ & NO & NA & NO \\
\hline Met/REC & TFDP1 & mut & TFDP1 & 2.40964 & $A$ & $\begin{array}{l}\text { filter } \\
\text { event_priority }\end{array}$ & gf - & NO & NA & NO \\
\hline Met/REC & ZBP1 & expr & $\begin{array}{l}\text { ZBP1 } \\
\text { ACAT2_cg2350 }\end{array}$ & 3.61446 & E & $\begin{array}{l}\text { filter } \\
\text { sample_freque }\end{array}$ & NA & NO & NA & NO \\
\hline Met/REC & ACAT2 & meth & $\begin{array}{l}0094 \\
\text { AGAP1_cg0880 }\end{array}$ & 7.22892 & NA & $\begin{array}{l}\text { ncy_filter } \\
\text { sample_freque }\end{array}$ & NA & NO & NA & NO \\
\hline Met/REC & AGAP1 & meth & $\begin{array}{l}5497 \\
\text { ALDOA_ALDOA }\end{array}$ & 6.0241 & NA & $\begin{array}{l}\text { ncy_filter } \\
\text { event_priority_ }\end{array}$ & NA & NO & NA & NO \\
\hline Met/REC & ALDOA & fmeth & $\begin{array}{c}\text { cg07025134 } \\
\overline{\text { AMH_cg266598 }}\end{array}$ & 2.40964 & C & $\begin{array}{l}\text { filter } \\
\text { sample_freque }\end{array}$ & $\begin{array}{l}\text { NA } \\
\text { camp_cyto }\end{array}$ & NO & NA & NO \\
\hline Met/REC & AMH & meth & $\begin{array}{l}\text { ASPSCR1_cg06 } \\
\text { ASP }\end{array}$ & 7.22892 & NA & $\begin{array}{l}\text { ncy_filter } \\
\text { sample_freque }\end{array}$ & kine_tgf & NO & NA & NO \\
\hline Met/REC & ASPSCR1 & meth & $\begin{array}{l}238300 \\
\text { BLNK_BLNK_cg }\end{array}$ & 6.0241 & NA & $\begin{array}{l}\text { ncy_filter } \\
\text { event_priority_ }\end{array}$ & NA & NO & NA & NO \\
\hline Met/REC & BLNK & fmeth & $\begin{array}{l}02980499 \\
\text { BRMS1_cg2158 }\end{array}$ & 1.20482 & C & $\begin{array}{l}\text { filter } \\
\text { sample_freque }\end{array}$ & NA & NO & NA & NO \\
\hline Met/REC & BRMS1 & meth & $\begin{array}{l}2163 \\
\text { CAV1_MIR107_ }\end{array}$ & 7.22892 & NA & $\begin{array}{l}\text { ncy_filter } \\
\text { event_priority_ }\end{array}$ & $\begin{array}{l}\text { NA } \\
\text { focal_adhe }\end{array}$ & NO & NA & NO \\
\hline Met/REC & CAV1 & fmeth & $\begin{array}{l}\text { cg24445034 } \\
\text { CCDC59_CCD } \\
\text { C59_cg0190390 }\end{array}$ & 1.20482 & C & $\begin{array}{l}\text { filter } \\
\text { event_priority_ }\end{array}$ & sion & YES & YES & YES \\
\hline Met/REC & CCDC59 & fmeth & CCND2 ca0380 & 1.20482 & C & samnle freque & $\begin{array}{l}\text { NA } \\
\text { cell_cycle_f } \\
\text { ocal_adhes } \\
\text { ion_jak_sta }\end{array}$ & YES & YES & NO \\
\hline Met/REC & CCND2 & meth & $\begin{array}{l}1902 \\
\text { CHAF1A_cg238 }\end{array}$ & 6.0241 & NA & $\begin{array}{l}\text { ncy_filter } \\
\text { sample_freque }\end{array}$ & _akt_wnt & NO & NA & NO \\
\hline Met/REC & CHAF1A & meth & 11775 & 9.63855 & NA & ncy_filter & NA & NO & NA & NO \\
\hline
\end{tabular}




\begin{tabular}{|c|c|c|c|c|c|c|c|c|c|c|}
\hline Met/REC & CHST12 & meth & $\begin{array}{l}\text { CHST12_cg101 } \\
45585 \\
\text { CRABP2_cg274 }\end{array}$ & 6.0241 & NA & $\begin{array}{l}\text { sample_freque } \\
\text { ncy_filter } \\
\text { sample_freque }\end{array}$ & NA & NO & NA & NO \\
\hline Met/REC & CRABP2 & meth & $\begin{array}{l}93997 \\
\text { CROCC_CROC }\end{array}$ & 6.0241 & NA & $\begin{array}{l}\text { ncy_filter } \\
\text { event_priority_ }\end{array}$ & NA & NO & NA & NO \\
\hline Met/REC & CROCC & fmeth & $\begin{array}{l}\text { C_cg23732962 } \\
\text { CYBA_CYBA_c }\end{array}$ & 1.20482 & C & $\begin{array}{l}\text { filter } \\
\text { event_priority_ }\end{array}$ & NA & NO & NA & NO \\
\hline Met/REC & CYBA & fmeth & $\begin{array}{l}\text { g24046 } 411 \\
\text { CYBA_CYBA_c }\end{array}$ & 1.20482 & C & $\begin{array}{l}\text { filter } \\
\text { event_priority_ }\end{array}$ & NA & NO & NA & NO \\
\hline Met/REC & CYBA & fmeth & $\begin{array}{l}\text { g2717 } 6 \overline{6} 614 \\
\text { DCAF12L1_cg0 }\end{array}$ & 1.20482 & C & $\begin{array}{l}\text { filter } \\
\text { sample_freque }\end{array}$ & NA & NO & NA & NO \\
\hline Met/REC & DCAF12L1 & meth & $\begin{array}{l}\text { 1913196 } \\
\text { DLG2_cg10901 }\end{array}$ & 4.81928 & NA & $\begin{array}{l}\text { ncy_filter } \\
\text { sample_freque }\end{array}$ & NA & NO & NA & NO \\
\hline Met/REC & DLG2 & meth & $\begin{array}{l}805 \\
\text { DPP9_cg24423 }\end{array}$ & 7.22892 & NA & $\begin{array}{l}\text { ncy_filter } \\
\text { sample_freque }\end{array}$ & NA & NO & NA & NO \\
\hline Met/REC & DPP9 & meth & $\begin{array}{l}897 \\
\text { DPYSL2_cg028 }\end{array}$ & 7.22892 & NA & $\begin{array}{l}\text { ncy_filter } \\
\text { sample_freque }\end{array}$ & NA & NO & NA & NO \\
\hline Met/REC & DPYSL2 & meth & $\begin{array}{l}35343 \\
\text { EGLN1_cg2001 }\end{array}$ & 7.22892 & NA & $\begin{array}{l}\text { ncy_filter } \\
\text { sample_freque }\end{array}$ & NA & NO & NA & NO \\
\hline Met/REC & EGLN1 & meth & $\begin{array}{l}5535 \\
\text { EIF5A_cg24699 }\end{array}$ & 7.22892 & NA & $\begin{array}{l}\text { ncy_filter } \\
\text { sample_freque }\end{array}$ & NA & YES & YES & YES \\
\hline Met/REC & EIF5A & meth & $\begin{array}{l}433 \\
\text { EIF5A2_EIF5A2 }\end{array}$ & 7.22892 & NA & $\begin{array}{l}\text { ncy_filter } \\
\text { event_priority_ }\end{array}$ & NA & NO & NA & NO \\
\hline Met/REC & EIF5A2 & fmeth & $\begin{array}{l}\text { cg12598803 } \\
\text { EIF5A2_EIF5A2 }\end{array}$ & 1.20482 & C & $\begin{array}{l}\text { filter } \\
\text { event_priority_ }\end{array}$ & NA & NO & NA & NO \\
\hline Met/REC & EIF5A2 & fmeth & $\begin{array}{l}\text { cg26209058 } \\
\bar{E} N 1 \text { cg027393 }\end{array}$ & 1.20482 & C & $\begin{array}{l}\text { filter } \\
\text { sample_freque }\end{array}$ & NA & NO & NA & NO \\
\hline Met/REC & EN1 & meth & $\begin{array}{l}46 \\
\text { EP400NL_EP40 } \\
\text { ONL_cg2563298 }\end{array}$ & 4.81928 & NA & $\begin{array}{l}\text { ncy_filter } \\
\text { event_priority }\end{array}$ & NA & NO & NA & NO \\
\hline Met/REC & EP400NL & fmeth & 3 & 1.20482 & C & & NA & NO & NA & NO \\
\hline Met/REC & ERBB2 & fmeth & $\begin{array}{l}\text { ERBB2_MIR125 } \\
\text { A_cg19782652 } \\
\text { ERBB2_MIR134 }\end{array}$ & 1.20482 & C & $\begin{array}{l}\text { event_priority_ } \\
\text { filter } \\
\text { event_priority_ }\end{array}$ & $\begin{array}{l}\text { ocal_adhes } \\
\text { ion } \\
\text { adherens_f }\end{array}$ & YES & YES & NO \\
\hline Met/REC & ERBB2 & fmeth & _cg26238975 & 1.20482 & C & filter & ocal_adhes & NO & NA & NO \\
\hline
\end{tabular}




\begin{tabular}{|c|c|c|c|c|c|c|c|c|c|c|}
\hline & & & & & & & ion & & & \\
\hline Met/REC & FBLN5 & meth & $\begin{array}{l}\text { FBLN5_cg2188 } \\
3802 \\
\text { FBN1_cg12975 }\end{array}$ & 7.22892 & NA & $\begin{array}{l}\text { sample_freque } \\
\text { ncy_filter } \\
\text { sample_freque }\end{array}$ & NA & NO & NA & NO \\
\hline Met/REC & FBN1 & meth & $\begin{array}{l}862 \\
\text { FBXO43_cg237 }\end{array}$ & 4.81928 & NA & $\begin{array}{l}\text { ncy_filter } \\
\text { sample_freque }\end{array}$ & NA & NO & NA & NO \\
\hline Met/REC & FBXO43 & meth & $\begin{array}{l}00125 \\
\text { FNTA_FNTA_cg }\end{array}$ & 9.63855 & NA & $\begin{array}{l}\text { ncy_filter } \\
\text { event_priority__ }\end{array}$ & NA & NO & NA & NO \\
\hline Met/REC & FNTA & fmeth & $\begin{array}{l}07294 \overline{2} 34 \\
\text { GALNT2_cg005 }\end{array}$ & 2.40964 & C & $\begin{array}{l}\text { filter } \\
\text { event_priority_ }\end{array}$ & NA & YES & YES & YES \\
\hline Met/REC & GALNT2 & meth & $\begin{array}{l}89617 \\
\text { GALNT2_cg005 }\end{array}$ & 6.0241 & $\mathrm{~F}$ & $\begin{array}{l}\text { filter } \\
\text { sample_freque }\end{array}$ & NA & NO & NA & NO \\
\hline Met/REC & GALNT2 & meth & $\begin{array}{l}89617 \\
\text { GDAP1_GDAP1 }\end{array}$ & 6.0241 & NA & $\begin{array}{l}\text { ncy_filter } \\
\text { event_priority_ }\end{array}$ & NA & NO & NA & NO \\
\hline Met/REC & GDAP1 & fmeth & $\begin{array}{l}\operatorname{cg} 221 \overline{05} 613 \\
\overline{G L R X 5} \text { (cg2600 }\end{array}$ & 2.40964 & C & $\begin{array}{l}\text { filter } \\
\text { sample_freque }\end{array}$ & NA & NO & NA & NO \\
\hline Met/REC & GLRX5 & meth & $\begin{array}{l}7049 \\
\text { GNB4_GNB4_c }\end{array}$ & 7.22892 & NA & $\begin{array}{l}\text { ncy_filter } \\
\text { event_priority_ }\end{array}$ & NA & NO & NA & NO \\
\hline Met/REC & GNB4 & fmeth & $\begin{array}{l}\text { g01750 } 654 \\
\text { HAUS5_HAUS5 }\end{array}$ & 1.20482 & C & $\begin{array}{l}\text { filter } \\
\text { event_priority_ }\end{array}$ & pi3k_akt & NO & NA & NO \\
\hline Met/REC & HAUS5 & fmeth & $\begin{array}{l}\text { cg05296832 } \\
\text { HEATR1_cg009 }\end{array}$ & 1.20482 & C & $\begin{array}{l}\text { filter } \\
\text { sample_freque }\end{array}$ & NA & NO & NA & NO \\
\hline Met/REC & HEATR1 & meth & $\begin{array}{l}07549 \\
\text { HNRNPM_HNR } \\
\text { NPM_cg059230 }\end{array}$ & 7.22892 & NA & $\begin{array}{l}\text { ncy_filter } \\
\text { event_priority_ }\end{array}$ & NA & NO & NA & NO \\
\hline Met/REC & HNRNPM & fmeth & $\begin{array}{l}62 \\
\text { HOMER3_HOM } \\
\text { ER3_cg197889 }\end{array}$ & 1.20482 & C & $\begin{array}{l}\text { filter } \\
\text { event_priority_ }\end{array}$ & NA & NO & NA & NO \\
\hline Met/REC & HOMER3 & fmeth & $\begin{array}{l}57 \\
\text { HTR3E_HTR3E }\end{array}$ & 1.20482 & C & $\begin{array}{l}\text { filter } \\
\text { event_priority }\end{array}$ & NA & NO & NA & NO \\
\hline Met/REC & HTR3E & fmeth & $\begin{array}{l}\text { cg11158819 } \\
\text { KLHL20_cg117 }\end{array}$ & 1.20482 & C & $\begin{array}{l}\text { filter } \\
\text { sample_freque }\end{array}$ & NA & NO & NA & NO \\
\hline Met/REC & KLHL20 & meth & $\begin{array}{l}20170 \\
\text { LBH_cg250724 }\end{array}$ & 7.22892 & NA & $\begin{array}{l}\text { ncy_filter } \\
\text { sample_freque }\end{array}$ & NA & NO & NA & NO \\
\hline Met/REC & LBH & meth & 36 & 7.22892 & NA & ncy_filter & NA & NO & NA & NO \\
\hline
\end{tabular}




\begin{tabular}{|c|c|c|c|c|c|c|c|c|c|c|}
\hline Met/REC & LMX1A & fmeth & $\begin{array}{l}\text { LMX1A_LMX1A } \\
\text { cg23971170 } \\
\text { LRRK1_cg2416 }\end{array}$ & 1.20482 & C & $\begin{array}{l}\text { event_priority_ } \\
\text { filter } \\
\text { sample_freque }\end{array}$ & NA & NO & NA & NO \\
\hline Met/REC & LRRK1 & meth & $\begin{array}{l}3563 \\
\text { MAGEA10_MA } \\
\text { GEA10_cg1996 }\end{array}$ & 6.0241 & NA & $\begin{array}{l}\text { ncy_filter } \\
\text { event_priority }\end{array}$ & NA & NO & NA & NO \\
\hline Met/REC & MAGEA10 & fmeth & $\begin{array}{l}4192 \\
\text { MAN2B1_cg024 }\end{array}$ & 1.20482 & C & $\begin{array}{l}\text { filter } \\
\text { sample_freque }\end{array}$ & NA & NO & NA & NO \\
\hline Met/REC & MAN2B1 & meth & $\begin{array}{l}84047 \\
\text { MAPK12_cg219 }\end{array}$ & 8.43373 & NA & $\begin{array}{l}\text { ncy_filter } \\
\text { sample_freque }\end{array}$ & NA & NO & NA & NO \\
\hline Met/REC & MAPK12 & meth & $\begin{array}{l}74239 \\
\text { MBP_cg009895 }\end{array}$ & 8.43373 & NA & $\begin{array}{l}\text { ncy_filter } \\
\text { sample_freque }\end{array}$ & mapk_vegf & NO & NA & NO \\
\hline Met/REC & MBP & meth & $\begin{array}{l}38 \\
\text { MED11_cg0983 }\end{array}$ & 6.0241 & NA & $\begin{array}{l}\text { ncy_filter } \\
\text { sample_freque }\end{array}$ & NA & NO & NA & NO \\
\hline Met/REC & MED11 & meth & $\begin{array}{l}2947 \\
\text { MORF4L1_MO } \\
\text { RF4L1_cg20905 }\end{array}$ & 8.43373 & NA & $\begin{array}{l}\text { ncy_filter } \\
\text { event_priority__ }\end{array}$ & NA & NO & NA & NO \\
\hline Met/REC & MORF4L1 & fmeth & $\begin{array}{l}746 \\
\text { NLGN2_cg1009 }\end{array}$ & 1.20482 & C & $\begin{array}{l}\text { filter } \\
\text { sample_freque }\end{array}$ & NA & NO & NA & NO \\
\hline Met/REC & NLGN2 & meth & $\begin{array}{l}2265 \\
\text { NOS3_cg12547 }\end{array}$ & 7.22892 & NA & $\begin{array}{l}\text { ncy_filter } \\
\text { sample_freque }\end{array}$ & $\begin{array}{l}\text { NA } \\
\text { pi3k_akt_v }\end{array}$ & NO & NA & NO \\
\hline Met/REC & NOS3 & meth & $\begin{array}{l}085 \\
\text { NR1H2_cg1467 }\end{array}$ & 7.22892 & NA & $\begin{array}{l}\text { ncy_filter } \\
\text { sample_freque }\end{array}$ & egf & NO & NA & NO \\
\hline Met/REC & NR1H2 & meth & $\begin{array}{l}3743 \\
\text { PCCB_cg05582 }\end{array}$ & 4.81928 & NA & $\begin{array}{l}\text { ncy_filter } \\
\text { sample_freque }\end{array}$ & NA & NO & NA & NO \\
\hline Met/REC & РССВ & meth & $\begin{array}{l}311 \\
\text { PCNT_PCNT_C }\end{array}$ & 7.22892 & NA & $\begin{array}{l}\text { ncy_filter } \\
\text { event_priority_ }\end{array}$ & NA & YES & YES & YES \\
\hline Met/REC & PCNT & fmeth & $\begin{array}{l}\text { g13822122 } \\
\text { PNMT_PNMT_c }\end{array}$ & 1.20482 & C & $\begin{array}{l}\text { filter } \\
\text { event_priority_ }\end{array}$ & NA & NO & NA & NO \\
\hline Met/REC & PNMT & fmeth & $\begin{array}{l}\text { g16268778 } \\
\text { PPP1R1B_PPP } \\
\text { 1R1B_cg06068 }\end{array}$ & 1.20482 & C & $\begin{array}{l}\text { filter } \\
\text { event_priority }\end{array}$ & NA & NO & NA & NO \\
\hline Met/REC & PPP1R1B & fmeth & $\begin{array}{l}801 \\
\text { PPP1R1B_PPP } \\
\text { 1R1B_cg09762 }\end{array}$ & 1.20482 & C & $\begin{array}{l}\text { filter } \\
\text { event_priority_ }\end{array}$ & camp & NO & NA & NO \\
\hline Met/REC & PPP1R1B & fmeth & 778 & 1.20482 & C & filter & camp & NO & NA & NO \\
\hline
\end{tabular}




\begin{tabular}{|c|c|c|c|c|c|c|c|c|c|c|}
\hline Met/REC & PRF1 & fmeth & $\begin{array}{l}\text { PRF1_PRF1_cg } \\
12336290 \\
\text { RBMS2_cg2300 }\end{array}$ & 1.20482 & C & $\begin{array}{l}\text { event_priority_ } \\
\text { filter } \\
\text { sample_freque }\end{array}$ & apoptosis & NO & NA & NO \\
\hline Met/REC & RBMS2 & meth & $\begin{array}{l}2907 \\
\text { RNF138_cg221 }\end{array}$ & 8.43373 & NA & $\begin{array}{l}\text { ncy_filter } \\
\text { sample_freque }\end{array}$ & NA & NO & NA & NO \\
\hline Met/REC & RNF138 & meth & $\begin{array}{l}41237 \\
\text { RPLP2_cg0148 }\end{array}$ & 4.81928 & NA & $\begin{array}{l}\text { ncy_filter } \\
\text { sample_freque }\end{array}$ & NA & NO & NA & NO \\
\hline Met/REC & RPLP2 & meth & $\begin{array}{l}5797 \\
\text { RPRD1A_RPR } \\
\text { D1A cg000198 }\end{array}$ & 6.0241 & NA & $\begin{array}{l}\text { ncy_filter } \\
\text { event priority }\end{array}$ & NA & YES & YES & YES \\
\hline Met/REC & RPRD1A & fmeth & $\begin{array}{l}09 \\
\text { RPRD1A_RPR } \\
\text { D1A_cg196297 }\end{array}$ & 1.20482 & C & $\begin{array}{l}\text { filter } \\
\text { event_priority_ }\end{array}$ & NA & NO & NA & NO \\
\hline Met/REC & RPRD1A & fmeth & $\begin{array}{l}10 \\
\text { RYBP_cg0 } 04421\end{array}$ & 1.20482 & C & $\begin{array}{l}\text { filter } \\
\text { sample_freque }\end{array}$ & NA & YES & YES & NO \\
\hline Met/REC & RYBP & meth & $\begin{array}{l}631 \\
\text { SLC45A4_cg08 }\end{array}$ & 7.22892 & NA & $\begin{array}{l}\text { ncy_filter } \\
\text { sample_freque }\end{array}$ & NA & NO & NA & NO \\
\hline Met/REC & SLC45A4 & meth & $\begin{array}{l}829393 \\
\text { SNUPN_cg2225 }\end{array}$ & 7.22892 & NA & $\begin{array}{l}\text { ncy_filter } \\
\text { sample_freque }\end{array}$ & NA & YES & YES & YES \\
\hline Met/REC & SNUPN & meth & $\begin{array}{l}8732 \\
\text { SORBS3_cg079 }\end{array}$ & 4.81928 & NA & $\begin{array}{l}\text { ncy_filter } \\
\text { sample_freque }\end{array}$ & NA & NO & NA & NO \\
\hline Met/REC & SORBS3 & meth & $\begin{array}{l}24703 \\
\text { SPIRE2_cg0740 }\end{array}$ & 6.0241 & NA & $\begin{array}{l}\text { ncy_filter } \\
\text { sample_freque }\end{array}$ & NA & NO & NA & NO \\
\hline Met/REC & SPIRE2 & meth & $\begin{array}{l}2062 \\
\text { SPOPL_SPOPL }\end{array}$ & 8.43373 & NA & $\begin{array}{l}\text { ncy_filter } \\
\text { event_priority_ }\end{array}$ & NA & NO & NA & NO \\
\hline Met/REC & SPOPL & fmeth & $\begin{array}{l}\text { cg09464263 } \\
\text { STIM1_cg04346 }\end{array}$ & 1.20482 & C & $\begin{array}{l}\text { filter } \\
\text { sample_freque }\end{array}$ & NA & NO & NA & NO \\
\hline Met/REC & STIM1 & meth & $\begin{array}{l}968 \\
\text { TAF1D_TAF1D }\end{array}$ & 6.0241 & NA & $\begin{array}{l}\text { ncy_filter } \\
\text { event_priority_ }\end{array}$ & NA & NO & NA & NO \\
\hline Met/REC & TAF1D & fmeth & $\begin{array}{l}\text { cg10353388 } \\
\text { TEX11_cg0816 }\end{array}$ & 1.20482 & C & $\begin{array}{l}\text { filter } \\
\text { sample_freque }\end{array}$ & NA & NO & NA & NO \\
\hline Met/REC & TEX11 & meth & $\begin{array}{l}7097 \\
\text { TNK2_cg03207 }\end{array}$ & 7.22892 & NA & $\begin{array}{l}\text { ncy_filter } \\
\text { sample_freque }\end{array}$ & NA & NO & NA & NO \\
\hline Met/REC & TNK2 & meth & $\begin{array}{l}310 \\
\text { TNPO1_cg0557 }\end{array}$ & 4.81928 & NA & $\begin{array}{l}\text { ncy_filter } \\
\text { sample_freque }\end{array}$ & NA & NO & NA & NO \\
\hline Met/REC & TNPO1 & meth & 9703 & 6.0241 & NA & ncy_filter & NA & NO & NA & NO \\
\hline
\end{tabular}




\begin{tabular}{|c|c|c|c|c|c|c|c|c|c|c|}
\hline Met/REC & TOLLIP & meth & $\begin{array}{l}\text { TOLLIP_cg0136 } \\
9233 \\
\text { TRRAP cg0345 }\end{array}$ & 6.0241 & NA & $\begin{array}{l}\text { sample_freque } \\
\text { ncy_filter } \\
\text { sample freque }\end{array}$ & NA & YES & YES & YES \\
\hline Met/REC & TRRAP & meth & $\begin{array}{l}7229 \\
\text { UMPS_cg15050 }\end{array}$ & 6.0241 & NA & $\begin{array}{l}\text { ncy_filtēer } \\
\text { sample_freque }\end{array}$ & NA & NO & NA & NO \\
\hline Met/REC & UMPS & meth & $\begin{array}{l}328 \\
\text { UST_cg212730 }\end{array}$ & 6.0241 & NA & $\begin{array}{l}\text { ncy_filter } \\
\text { sample_freque }\end{array}$ & NA & YES & YES & YES \\
\hline Met/REC & UST & meth & $\begin{array}{l}29 \\
\text { WDR66_cg0176 }\end{array}$ & 6.0241 & NA & $\begin{array}{l}\text { ncy_filter } \\
\text { sample_freque }\end{array}$ & NA & NO & NA & NO \\
\hline Met/REC & WDR66 & meth & $\begin{array}{l}5077 \\
\text { ZFHX3_cg0570 }\end{array}$ & 6.0241 & NA & $\begin{array}{l}\text { ncy_filter } \\
\text { sample_freque }\end{array}$ & NA & NO & NA & NO \\
\hline Met/REC & ZFHX3 & meth & $\begin{array}{l}4496 \\
\text { ZIC3_ZIC3_cg1 }\end{array}$ & 6.0241 & NA & $\begin{array}{l}\text { ncy_filtēer } \\
\text { event_priority_ }\end{array}$ & NA & NO & NA & NO \\
\hline Met/REC & ZIC3 & fmeth & $\begin{array}{l}0451 \overline{7} 60 \\
\text { ZNF121_cg069 }\end{array}$ & 1.20482 & C & $\begin{array}{l}\text { filter } \\
\text { sample_freque }\end{array}$ & NA & NO & NA & NO \\
\hline Met/REC & ZNF121 & meth & $\begin{array}{l}98238 \\
\text { ZNF410_ZNF41 }\end{array}$ & 6.0241 & NA & $\begin{array}{l}\text { ncy_filter } \\
\text { event_priority_ }\end{array}$ & NA & YES & YES & YES \\
\hline Met/REC & ZNF410 & fmeth & $\begin{array}{l}\text { 0_cg12152256 } \\
\text { ZNF490_cg240 }\end{array}$ & 1.20482 & C & $\begin{array}{l}\text { filter } \\
\text { sample_freque }\end{array}$ & NA & NO & NA & NO \\
\hline Met/REC & ZNF490 & meth & $\begin{array}{l}93429 \\
\text { ZNF497_ZNF49 }\end{array}$ & 6.0241 & NA & $\begin{array}{l}\text { ncy_filter } \\
\text { event_priority_ }\end{array}$ & NA & YES & YES & YES \\
\hline Met/REC & ZNF497 & fmeth & $\begin{array}{l}\text { 7_cg14989522 } \\
\text { ZNF516_cg162 }\end{array}$ & 1.20482 & C & $\begin{array}{l}\text { filter } \\
\text { event_priority_}\end{array}$ & NA & NO & NA & NO \\
\hline Met/REC & ZNF516 & meth & $\begin{array}{l}75118 \\
\text { ZNF516_cg162 }\end{array}$ & 9.63855 & $\mathrm{~F}$ & $\begin{array}{l}\text { filter } \\
\text { sample_freque }\end{array}$ & NA & NO & NA & NO \\
\hline Met/REC & ZNF516 & meth & $\begin{array}{l}75118 \\
\text { ZNF580_cg262 }\end{array}$ & 9.63855 & NA & $\begin{array}{l}\text { ncy_filter } \\
\text { sample_freque }\end{array}$ & NA & NO & NA & NO \\
\hline Met/REC & ZNF580 & meth & $\begin{array}{l}09676 \\
\text { ZNF581_cg262 }\end{array}$ & 4.81928 & NA & $\begin{array}{l}\text { ncy_filter } \\
\text { sample_freque }\end{array}$ & NA & NO & NA & NO \\
\hline Met/REC & ZNF581 & meth & $\begin{array}{l}09676 \\
\text { ZNF791_cg240 }\end{array}$ & 4.81928 & NA & $\begin{array}{l}\text { ncy_filter } \\
\text { sample_freque }\end{array}$ & NA & NO & NA & NO \\
\hline Met/REC & ZNF791 & meth & 93429 & 6.0241 & NA & ncy_filter & NA & YES & YES & YES \\
\hline
\end{tabular}

